\title{
AUTOMORPHISMS AND ISOMORPHISMS OF REAL HENSELIAN FIELDS
}

\author{
RON BROWN
}

\begin{abstract}
Let $K$ and $L$ be ordered algebraic extensions of an ordered field $F$. Suppose $K$ and $L$ are Henselian with Archimedean real closed residue class fields. Then $K$ and $L$ are shown to be $F$-isomorphic as ordered fields if they have the same value group. Analogues to this result are proved involving orderings of higher level, unordered extensions, and, when $K$ and $L$ are maximal valued fields, transcendental extensions. As a corollary, generalized real closures at orderings of higher level are shown to be determined up to isomorphism by their value groups. The results on isomorphisms are applied to the computation of automorphism groups of $K$ and to the study of the fixed fields of groups of automorphisms of $K$. If $K$ is real closed and maximal with respect to its canonical valuation, then these fixed fields are shown to be exactly those real closed subfields of $K$ which are topologically closed in $K$. Generalizations of this fact are proved. An example is given to illustrate several aspects of the problems considered here.
\end{abstract}

1. Introduction. Throughout this section $K$ and $L$ denote valued field extensions of a valued field $F$. Suppose $K$ and $L$ are Henselian with Archimedean real closed residue class fields. Such field extensions arise in many contexts; examples are ultracompletions at real Harrison primes [HW], ultracompletions (or Henselizations) at real extended absolute values [Br3, $\S \S 1$ and 4], completions at 0 -primes [V], real closures of fields $[\mathbf{B}, \mathbf{A S}]$, generalized real closures at orderings of higher level [B , §5], and minimal ordered field extensions satisfying Rolle's Theorem for polynomials [BCP]. We study here the question of when $K$ and $L$ are $F$-isomorphic, and apply our results to the study of automorphisms of $K$.

We begin with an instructive example.

1.1 EXAMPLE. Let $F=\mathbf{R}((x))$, the field of Laurent series over the field of real numbers, $\mathbf{R}$. Set $K=F\left[x^{1 / 2}\right]$ and $L=F\left[(-x)^{1 / 2}\right]$. Then $K$ and $L$ are not $F$-isomorphic because $x \in F \cap K^{2}$ but $x \notin L^{2}$. If we identify the value group of $F$ for the $x$-adic valuation with $\mathbf{Z}$, then both $K$ and $L$ have value group $\frac{1}{2} \mathbf{Z}$. All three fields have residue class field isomorphic to $\mathbf{R}$.

The above example shows that $K$ and $L$ need not be $F$-isomorphic even though they induce isomorphic residue class field extensions of the residue class field of $F$ and isomorphic value group extensions of the value group of $F$. For ordered field extensions, however, the situation is simpler.

Let $v K, v L$ and $v F$ denote the value groups of $K, L$ and $F$.

Received by the editors April 7, 1987.

1980 Mathematics Subject Classification. Primary 12F10, 12F20, 12J10, $12 J 15$.

Partially supported by the National Science Foundation. 
1.2 THEOREM. Suppose $K$ and $L$ are algebraic extensions of $F$ with $v K=v L$. Suppose $S$ and $T$ are orderings of $K$ and $L$, respectively, with $S \cap F=T \cap F$. Then there exists a unique $F$-isomorphism $\Delta: K \rightarrow L$ with $\Delta(S)=T$.

In the statement of the above theorem we are implicitly assuming that the value groups of $K$ and $L$ have been canonically identified with subgroups of a divisible hull of the value group of $F$. (Recall that $K$ and $L$ are algebraic valued field extensions of $F$, so $v K / v F$ and $v L / v F$ are torsion.)

Note that Theorem 1.2 includes the classical Artin-Schreier theorem that there is a unique $F$-isomorphism between any two real closures of $F$ inducing the same ordering on $F$. (For such real closures $K$ and $L$, the value groups of $K$ and $L$ are both equal to the divisible hull of the value group of $F$.) Theorem 1.2 will be proved in $\S 3$ as a consequence of a more general embedding theorem. Applications of Theorem 1.2 given in $\S 3$ include necessary and sufficient conditions for the $F$ isomorphism of (unordered) algebraic field extensions $K$ and $L$ of $F$ (e.g., that $v K=v L$ and $\left.K^{2} \cap F=L^{2} \cap F\right)$, computations of the automorphism group of an algebraic extension $K / F$ (it is isomorphic to $\operatorname{Hom}(v K / v F, \mathbf{Z} / 2 \mathbf{Z})$ ), and criteria for the existence of extensions of homomorphisms $F \rightarrow K$ to automorphisms of $K$.

In $\S 4$ we prove analogues of the main results of $\S 3$ for field extensions $K / F$ and $L / F$ which are not necessarily algebraic, under the hypothesis that $K$ and $L$ are maximal valued fields with Archimedean real closed residue class fields. For example, here is an isomorphism theorem for unordered fields.

1.3 THEOREM. Suppose $K$ and $L$ are maximal valued fields with isomorphic Archimedean real closed residue class fields. Suppose $K^{2} \cap F=L^{2} \cap F$ and that there is an isomorphism of ordered groups $\Psi: v K \rightarrow v L$ fixing $v F$. Let $v_{K}$ and $v_{L}$ denote the valuations on the valued fields $K$ and $L$. Then there is an $F$-isomorphism $\Delta: K \rightarrow L$ with $v_{L} \Delta=\Psi v_{K}$.

In $\S 5$ we generalize Theorem 1.2 and its analogue in $\S 4$ for maximal valued extensions by allowing orderings of higher level [B] as well as the classical orderings of the Artin-Schreier theory. Example 1.1 illustrates that some restriction will be needed on the exact levels of the orderings of higher level (as Becker has observed for these fields, every ordering of two power level of $F$ extends to $K$ and to $L$ ). In that section we will review and develop some of the basic theory of orderings of higher level, with special focus on the "2-primary component" of an ordering of higher level. As a corollary we obtain the following.

1.4 THEOREM. Suppose $P$ is an ordering of higher level of $F$ and that $(K, T)$ and $(L, S)$ are generalized real closures of $(F, P)$. If $v K=v L$, then there is a unique $F$-isomorphism from $K$ to $L$.

The isomorphism theorems of $\S 3$ and $\S 4$ are applied in $\S \S 6$ and 7 to study the automorphisms of $K$. We have the usual bijective Galois correspondence between the set of fixed fields of $K$ with respect to groups of automorphisms of $K$ and the set of groups of automorphisms of $K$ of the form Aut $(K / E)$ where $E$ is a subfield of $K$. Our main effort here is directed toward characterizing intrinsically the fixed fields of $K$ with respect to sets of automorphisms of $K$ (abbreviated, "fixed subfields of $K$ "). It is easy to check (Proposition 6.1) that such a fixed subfield is topologically closed in $K$ (with respect to the interval topology for any ordering on $K$-all these 
topologies coincide) and that its relative algebraic closure in $K$ is a multiquadratic extension. The converse is false, but not if $K$ is a maximal valued field.

1.5 THEOREM. Suppose $K$ is a maximal valued field with Archimedean real closed residue class field. Then a subfield $F$ of $K$ is a fixed subfield if and only if $F$ is topologically closed in $K$ and the relative algebraic closure of $F$ in $K$ is a multiquadratic extension of $F$.

For example the field of real algebraic numbers is a fixed subfield of any nonarchimedean real closed field which is a maximal valued field for its Archimedean class valuation. Theorem 1.5 takes a simpler form if $K$ is real closed; any fixed subfield in this case is actually algebraically closed in $K$.

In $\S 7$ we show that if the extension $K / F$ has transcendence degree one and $F$ is topologically and algebraically closed in $K$, then $F$ is a fixed subfield of $K$. This fact is proved as a corollary of a computation of the group of valued field automorphisms of $K$ which fix $F$. We have no counterexamples to the assertion that the above fact is valid without the restriction on transcendence degree. However, we do give in $\S 7$ an example to show that the hypothesis that $K$ is a maximal valued field cannot be dropped from Theorem 1.5.

In $\S 8$ we consider a more elaborate example of a maximal valued field $K$ with Archimedean real closed residue class field and with infinite transcendence degree over a subfield $F$. This work is partly motivated by a feeling that the detailed consideration of nontrivial examples may be necessary for progress on the more intractable problems in this area. We compute the full automorphism group of $K / F$, and show that the subgroups of $\operatorname{Aut}(K / F)$ of the form $\operatorname{Aut}(K / S)$ where $K \supset S \supset F$ are exactly the closed subgroups of $\operatorname{Aut}(K / F)$ with respect to a natural topology (not the Krull topology). We show the algebraic closure in $K$ of a fixed subfield of $K$ need not be fixed. A second example is constructed showing that Theorem 1.5 fails if $K$ is not required to be a maximal valued field. A key feature of this example and of that in $\S 7$ is the construction of subsets of $K$ algebraically independent over $F$.

Some concepts and notation are introduced in $\S 2$ which are used throughout the paper (cf., 2.1 and 2.3). In particular we introduce the notions of "real Henselian fields" and "real Henselizations". These concepts can be set into a general theory of Henselizations with respect to extended absolute values $[\mathbf{B r 3}, \S 4 ; \mathbf{B r 7}]$. We develop in $\S 2$, however, only the results which are needed in later sections. The main result is a universal property for real Henselizations. This material closely parallels the theory of Henselian valued fields, and none of it is surprising or difficult.

We end this introduction with a few comments on format and notation. First, at the beginning of each section we give covering hypotheses for that section. The reader is reminded that these hypotheses are not repeated in the statements of theorems in the section. Next, we regard a field extension $K / F$ as a field homomorphism $F \rightarrow K$ and we need this point of view in the proofs of a few theorems. In the statements of theorems and in most proofs, however, we implicitly assume that our field extensions are given by inclusion maps.

We use standard notations. $\mathbf{N}, \mathbf{Z}, \mathbf{Q}$, and $\mathbf{R}$ denote the sets of positive integers, integers, rational numbers, and real numbers, respectively. $R^{\cdot}$ denotes the group of multiplicative units of a unitary ring $R$ (so $\mathbf{Z}=\{1,-1\}$ ). " $A \backslash B$ " denotes the 
complement of the set $B$ in the set $A$, and " $f \mid A$ " denotes the restriction of a map $f$ to a subset $A$ of its domain. Less standard is our notation " $E\left(\left(t^{\Gamma}\right)\right)$ " for the field of generalized power series (perhaps more correctly called generalized Laurent series) with coefficient field $E$ and valued group $\Gamma$ (and trivial factor set). This field is variously denoted elsewhere by $S(E, \Gamma, 1)$ [S, p. 23], $E((t))^{\Gamma}[\mathbf{R}$, p. 103], $S(\Gamma, E)$ [Bo, p. 174] and $E\left(t^{\Gamma}\right)$ [K, p. 314]. The definition of $E\left(\left(t^{\Gamma}\right)\right)$ is reviewed in $\S 8$, where such a field is studied in detail.

2. Real Henselian fields. Throughout this section $K / F$ denotes a field extension. We further assume that $K$ is "real Henselian" in the sense of the following definition.

2.1 DEFINITION. $K$ is real Henselian if it admits a Henselian valuation with Archimedean real closed residue class field. $K$ is a real Henselization of $F$ if it admits a Henselian valuation, say $v$, with Archimedean real closed residue class field, $K / F$ is algebraic, and $v\left(K^{*}\right)=v\left(F^{*}\right)$.

Examples of real Henselian fields were listed in the first paragraph of $\S 1$.

The above definitions of "real Henselian" and "real Henselization" are strictly of the quick-and-dirty variety. A much more general and conceptual treatment is possible, closely mimicing the corresponding theory for valuations. See [Br3, §4] and $[\mathbf{B r} \mathbf{7}]$ for work in this direction. We develop in this section only facts that will be needed later in the paper.

2.2 Proposition. $K$ admits a unique place into $\mathbf{R}$, say $\sigma$, and the associated valuation, call it $v$, is the unique (up to equivalence) valuation on $K$ with Archimedean real closed residue class field. Every ordering on $K$ induces $\sigma$ and $v$, and $\sigma \tau=\sigma$ and $v \tau$ is equivalent to $v$ for any automorphism $\tau$ of $K$.

Recall that an ordering $P$ of $K$ "induces" a place $\sigma: K \rightarrow \mathbf{R} \cup\{\infty\}$ if and only if $\sigma(P) \geq 0[\mathbf{B r} \mathbf{1}]$; an equivalent condition is that

$$
\sigma^{-1}\left(\mathbf{R}^{\cdot}\right) \cap P=\sigma^{-1}\left(\mathbf{R}^{\cdot 2}\right) \text {. }
$$

The valuation "induced" by $P$ is the Archimedean class valuation .

PROOF OF 2.2. Let $v$ be the Henselian valuation on $K$ with Archimedean real closed residue class field. Since any Archimedean ordered field has an embedding into $\mathbf{R}, v$ is induced by a place, say $\sigma$, from $K$ into $\mathbf{R}$. If $\rho$ is any place from $K$ into $\mathbf{R}$ with $\rho \neq \sigma$, then there exists $a \in K$ with $\rho(a)<0$ and $\infty>\sigma(a)>0[\mathbf{B r} 2$, Theorem 2.1A]. Then our hypotheses imply that $a \in K^{2}$. This contradicts that $\rho(a)<0$. Thus $\sigma$ is the unique place from $K$ into $\mathbf{R}$. The other assertions of 2.2 follow easily from this one.

2.3 Notation. Say $E$ is a real Henselian field. We let $\sigma_{E}$ denote the unique place from $E$ into $\mathbf{R}$, and let $v_{E}$ (or just $v$ ) denote the associated valuation. For any $A \subset E$, we let $v A$ (or $v(A)$ ) denote $v\left(A \cap E^{*}\right)$. Finally we let $\bar{E}$ denote the residue class field of $v$ and $\bar{e}$ denote the residue class of any $e \in E$ with $v(e) \geq 0$.

We include the next proposition for completeness; it is well known.

2.4 LEMmA. Say $F$ is algebraically closed in $K$ and $K$ is real closed. Then $F$ is real closed.

ProOF. Say $a+b i \in K[i]$ (where $i^{2}=-1$ and $a, b \in K$ ) is algebraic over $F$. Then $a-b i$ is clearly algebraic over $F$, and so $a$ and $b$ are algebraic over $F$. We 
can conclude that $F[i]$ is algebraically closed in $K[i]$. Hence $F[i]$ is algebraically closed-so $F$ is real closed ([AS, Satz 4] or [L, Theorem 2.5]).

2.5 Proposition. For any integer $n>0, v\left(K^{n} \cap F\right)=v\left(K^{n}\right) \cap v(F)$.

Proof. Let $a \in K$ have $v\left(a^{n}\right) \in v F$. Then $n v(a)=v(b)$ for some $b \in F$. We may assume $\sigma_{K}\left(a^{n} / b\right)>0$ (if necessary replace $b$ by $-b$ ). Then $a^{n} / b=d^{n}$ for some unit $d \in K$ (recall that $K$ is real Henselian, cf. 2.1). Then $(a / d)^{n}=b$, so $n v(a)=v\left((a / d)^{n}\right) \in v\left(K^{n} \cap F\right)$. Thus $v\left(K^{n} \cap F\right) \supset v\left(K^{n}\right) \cap v(F)$. The reverse inclusion is obvious.

2.6 Proposition. Suppose $F$ has no proper algebraic extension in $K$. Then $F$ is real Henselian. Further, $v K / v F$ is torsion-free and $\bar{F}$ has no proper algebraic extension in $\bar{K}$.

Proof. Let $a$ be in the valuation ring $\sigma_{K}^{-1}(\mathbf{R})$; suppose $\bar{a}$ is algebraic over $\bar{F}$. Then there exist $b_{i} \in \sigma_{K}^{-1}(\mathbf{R}) \cap F$ with $\sum \bar{b}_{i} \bar{a}^{i}=0$ and with $\sum \bar{b}_{i} x^{i}$ an irreducible polynomial over $\bar{F}$. Since $K$ is a Henselian valued field, there exists $c \in K$ with $\bar{c}=\bar{a}$ and $\sum b_{i} c^{i}=0$. Then by hypothesis, $c \in F$, so $\bar{a} \in \bar{F}$. Thus $\bar{F}$ is algebraically closed in $\bar{K}$. Hence by Lemma $2.4, \bar{F}$ is real closed. $F$ is a Henselian valued field since it is algebraically closed in the Henselian field $K$ (after all, it has a Henselian algebraic extension in $K$ by $[\mathbf{E}, 17.10 \mathrm{~b}]$ ). Thus $F$ is real Henselian (Definition 2.1). That $v K / v F$ is torsion-free follows from the previous proposition and the observation that by hypothesis we have $K^{n} \cap F=F^{n}$ for any integer $n>0$.

2.7 Proposition. Let $\sigma$ be a place from $F$ into $\mathbf{R}$. Then there exists a real Henselization $E$ of $F$ such that $\sigma_{E}$ extends $\sigma$.

Proof. Let $E_{0}$ be a Henselization of the valued field $F$ (where the valuation is that induced by $\sigma$ ). Let $L$ be a real closure of $\bar{E}_{0}$ at the ordering induced by $\sigma$. Now take $E$ to be an unramified algebraic extension of $E_{0}$ with residue class field $L$ [R, p. 164, Proposition 3 (Mac Lane)].

We now show that real Henselizations satisfy a universal property which, in a more systematic treatment, might be used to define them.

2.8 Proposition. $K$ contains a unique real Henselization of $F$. More generally, if $E$ is a real Henselization of $F$ such that $\sigma_{K}$ and $\sigma_{E}$ have the same restriction to $F$, then there exists a unique $F$-homomorphism $\theta: E \rightarrow K$.

ProOF. Let $K_{0}$ denote the unique Henselization of $F$ in $K$ with respect to $v_{K} \mid F\left[\mathbf{E}\right.$, p. 131]. An algebraic extension $K_{1}$ of $F$ in $K$ is a real Henselization of $F$ if and only if $v K_{1}=v F, K_{1} \supset K_{0}$ (equivalently, $K_{1}$ is Henselian with respect to $v_{K} \mid K_{1}$ ), and $\bar{K}_{1}$ is the algebraic closure of $\bar{F}$ in $\bar{K}$ (equivalently, $\bar{K}_{1}$ is real closed, c.f. Proposition 2.4). The existence and uniqueness of such an extension $K_{1}$ are easy consequences of Hensel's Lemma, together with [R, p. 236, Theorem 2]. The remaining assertions of 2.8 follow from the uniqueness properties of ordinary Henselizations [E] and from [S, p. 219, Lemma 20].

2.9 Proposition. Say $H$ is a set of automorphisms of $K$. Then the fixed field $K^{H}$ is real Henselian.

Proof. Let $v=v_{K}$. By Proposition 2.6 we may assume $K / K^{H}$ is algebraic (otherwise replace $K$ by the algebraic closure of $K^{H}$ in $K$ and $H$ by the set of 
restrictions of elements of $H$ to this relative algebraic closure). $\overline{K^{H}}$ is the fixed field of $\bar{K}$ under the set of automorphisms of $\bar{K}$ induced by elements of $H[\mathbf{E}$, Theorems 19.6 and 19.12]. But these induced automorphisms are all trivial since $\bar{K}$ is an Archimedean real closed field. Thus $\overline{K^{H}}=\bar{K}$ is Archimedean real closed. It remains to show that $K^{H}$ is Henselian with respect to $v \mid K^{H}$. Let $K^{\prime}$ be an algebraic closure of $K$. Let $v^{\prime}$ and $w$ be extensions to $K^{\prime}$ of $v$ and $v \mid K^{H}$, respectively. It suffices to show that $v^{\prime}=w$. Since $K$ is Henselian, it suffices to show that $v=w \mid K$. There exists $\tau \in \operatorname{Aut}\left(K^{\prime} / K^{H}\right)$ with $w=v^{\prime} \tau$. Since $K / K^{H}$ is normal, $\tau \mid K \in \operatorname{Aut}\left(K / K^{H}\right)$. Thus $w|K=v \tau| K$. Hence by Proposition 2.2, $v=v \tau|K=w| K$, as required.

Our last proposition is a corollary of a theorem of Ostrowski [R, p. 236, Theorem 2]; we include it here for the convenience of the reader since we will use it several times.

2.10 PROPOSITION. K has no immediate proper algebraic extensions.

In 2.10 we of course mean immediate extensions with respect to the valuation $v_{K}$.

3. Isomorphisms of algebraic extensions. Throughout this section $K$ and $L$ will denote real Henselian algebraic extensions of a field $F$ such that $v_{K}$ and $v_{L}$ have the same restriction to $F$. Thus, we can and do canonically identify $v K$ and $v L$ with subgroups of some divisible hull of $v F$. (For notation and terminology see 2.1 and 2.3.)

3.1 THEOREM. Let $P_{K}$ and $P_{L}$ be orderings of $K$ and $L$, respectively, which induce the same ordering on $F$. Suppose $v K \subset v L$. Then there is a unique $F$ homomorphism $\Delta: K \rightarrow L$ with $\Delta\left(P_{K}\right) \subset P_{L}$. If $v K=v L$, then $\Delta$ is an isomorphism.

Proof. Let $K^{\prime}$ and $L^{\prime}$ denote real closures of $K$ and $L$ with respect to the orderings $P_{K}$ and $P_{L}$, respectively. Then $K^{\prime}$ and $L^{\prime}$ are real closures of $F$ with respect to $P_{K} \cap F$, so there exists an $F$-isomorphism $\theta: K^{\prime} \rightarrow L^{\prime}$. Now suppose $\alpha \in v K$, say with $n \alpha \in v F$ for some integer $n>0$. Then by Proposition 2.5 (and our hypothesis) there exists $a \in P_{K}$ and $b \in P_{L}$ with $v(a)=v(b)=\alpha$ and $a^{n} \in F$ and $b^{n} \in F$. Then

$$
(\theta(a) / b)^{n}=a^{n} / b^{n} \in P_{L} \cap \sigma_{L}^{-1}\left(\mathbf{R}^{\cdot}\right) \subset \sigma_{L}^{-1}\left(\mathbf{R}^{\cdot 2}\right) \subset L^{n} .
$$

Hence $\theta(a) / b \in L$ (all roots of unity in $L^{\prime}$ are also in $L$ since $L^{\prime}$ is formally real). Thus $\theta(a) \in L$. Thus $\theta$ maps a subfield $K_{0}$ of $K$ with $K_{0} \supset F$ and $v K_{0}=v K$ into $L$. $K_{0}$ has a real Henselization $K_{1}$ in $K$ and $\theta\left(K_{1}\right) \subset L$ (Proposition 2.8). Clearly, $K / K_{1}$ is an immediate extension $(\bar{K}$ is an algebraic extension of the real closed field $\bar{K}_{1}$ ). Thus $K=K_{1}$ (Proposition 2.10). Hence $\theta(K) \subset L$. Now simply take $\Delta=\theta \mid K$. That $\Delta\left(P_{K}\right) \subset P_{L}$ follows from the fact that $\theta\left(K^{\prime 2}\right) \subset L^{\prime 2}$. The uniqueness of $\Delta$ follows immediately from the uniqueness of the $F$-isomorphism between real closures of an ordered field. Finally, if $v K=v L$, then $L / \theta(K)$ is immediate, so $\Delta$ is an isomorphism (Proposition 2.10).

3.2 THEOREM. The following statements are equivalent.

(1) $K$ and $L$ are $F$-isomorphic as fields. 
(2) $K$ and $L$ are $F$-isomorphic as valued fields.

(3) $K^{n} \cap F=L^{n} \cap F$ for all integers $n>0$.

(4) $K^{2} \cap F=L^{2} \cap F$ and $v K=v L$.

(5) Some ordering of $F$ extends to an ordering of $K$ and to an ordering of $L$, and $v K=v L$.

ProOF. It suffices to establish the chain of implications $(2) \Rightarrow(1) \Rightarrow(3) \Rightarrow$ $(4) \Rightarrow(5) \Rightarrow(2)$. The first two implications are obvious and the last follows from the previous theorem. Now suppose (3) holds; we prove (4). We need only show $v K=v L$.

Let $\alpha \in v K$. Then $n \alpha \in v F$ for some $n>0$. Thus $\alpha=v(a)$ for some $a \in K$ with $a^{n} \in F$ (Proposition 2.5). Hence $a^{n} \in L^{n} \cap F$ by hypothesis. Say $b \in L$ has $b^{n}=a^{n}$. Then $\alpha=v(b) \in v L$. Then $v K \subset v L$. Equality follows by symmetry.

Now suppose (4) holds. Let $T$ be any ordering of $K$. We claim that $T \cap F$ extends to $L$. Just suppose $-1 \in(T \cap F) L^{2}$. Then $-1=a b^{2}$ for some $a \in T \cap F, b \in L$. Thus

$$
-a^{-1}=b^{2} \in(-T) \cap\left(F \cap L^{2}\right)=(-T) \cap\left(F \cap K^{2}\right) \subset T \cap(-T)=\{0\} .
$$

This contradiction shows that $-1 \notin(T \cap F) L^{2}$. But $L$ is superpythagorean [Br4, Corollary 8] and so $(T \cap F) L^{2}$ is contained in an ordering of $L$. This shows (5) holds. The theorem is proved.

3.3 REMARK. The covering hypothesis for this section, that $v_{K}$ and $v_{L}$ have the same restriction to $F$, is not necessary and is included only in order to make Theorems 3.1 and 3.2 easy to formulate. After all, $v_{K}$ and $v_{L}$ will have equivalent restrictions to $F$ if $\sigma_{K}\left|F=\sigma_{L}\right| F$, and this equality follows from any of the following conditions (cf., Theorems 3.1 and 3.2):

(A) some ordering of $F$ extends to an ordering of $K$ and to an ordering of $L$ (note by Proposition 2.2 that the extended orderings must induce $\sigma_{K}$ and $\sigma_{L}$ );

(B) $K^{2} \cap F=L^{2} \cap F$ (note that if $\sigma_{K}\left|F \neq \sigma_{L}\right| F$ then there exists $a \in F$ with $0>\sigma_{K}(a)$ and $\infty>\sigma_{L}(a)>0\left[\mathrm{Br2}\right.$, Theorem 2.1A], so that $a \notin K^{2}$ but $\left.a \in L^{2}\right)$;

(C) $K$ and $L$ are $F$-isomorphic (cf., Proposition 2.2).

Much effort has gone into developing for formally $p$-adic fields a theory analogous to that of formally real fields (e.g., see [PR, p. 1]). The next result reverses this pattern: it is an analogue for formally real fields of a result of Prestel and Roquette on formally $p$-adic fields [PR, Theorem 3.12]. In [Br7] we give a framework for a unified development of both theories. Theorem 3.2 generalizes fully (all five parts) to this broader context. The generalization includes the "isomorphism theorem for algebraic extensions" of $p$-valued fields [PR, Theorem 3.11].

3.4 THEOREM. Two real Henselian intermediate fields $E$ and $E^{\prime}$ of the extension $K / F$ are equal if and only if $v E=v E^{\prime}$.

Proof. Say $v E=v E^{\prime}$. Let $T$ be any ordering of $K$. By Theorem 3.1 there is a unique $F$-isomorphism $\theta: E \rightarrow E^{\prime}$ with $\theta(E \cap T)=E^{\prime} \cap T$. Again by $3.1, \theta$ extends to an automorphism $\theta^{\prime}$ of $K$ with $\theta^{\prime}(T)=T$. By 3.1, $\theta^{\prime}$ is the only $F$-automorphism of $K$ with $\theta^{\prime}(T)=T$. But then $\theta^{\prime}$ is the identity map on $K$ and so $E^{\prime}=\theta^{\prime}(E)=E$. The converse is trivial.

We now apply Theorem 3.1 to study $\operatorname{Aut}(K / F)$, the group of $F$-automorphisms of $K$. 
3.5 THEOREM. (A) There is a well-defined group isomorphism $\Phi: \operatorname{Aut}(K / F)$ $\rightarrow \operatorname{Hom}\left(v K / v F, \mathbf{Z}^{*}\right)$ such that for each $\tau \in \operatorname{Aut}(K / F)$ and $a \in K^{*}$ we have

$$
\Phi(\tau)(v(a)+v F)=\operatorname{sign}\left(\sigma_{K}\left(a / \tau^{-1}(a)\right)\right)
$$

(where sign: $\mathbf{R}^{\cdot} \rightarrow \mathbf{Z}^{\cdot}$ is the homomorphism with kernel $\mathbf{R}^{\cdot 2}$ ).

(B) Let $T$ be an ordering of $K$. The correspondence $\Psi: \tau \mapsto \tau(T)$ carries $\operatorname{Aut}(K / F)$ bijectively onto the set of orderings of $K$ containing $T \cap F$.

PROOF. Let $X_{T}$ be the set of orderings of $K$ containing $T \cap F$. That $\Psi$ : $\operatorname{Aut}(K / F) \rightarrow X_{T}$ is a bijection follows directly from Theorem 3.1. Let

$$
\varphi_{T}: X_{T} \rightarrow \operatorname{Hom}\left(v K / v F, \mathbf{Z}^{*}\right)
$$

be the bijection with

$$
\varphi_{T}(S)(v(a)+v F)=\operatorname{sgn}_{T}(a) \operatorname{sgn}_{S}(a)
$$

for all $S \in X_{T}, a \in K^{\bullet}$ (where $\operatorname{sgn}_{T}: K^{\bullet} \rightarrow \mathbf{Z}^{\bullet}$ is the signature homomorphism; the kernel of $\operatorname{sgn}_{T}$ is $T^{*}$ ) [Br1, last paragraph, p. 635].

For all $\tau \in \operatorname{Aut}(K / F)$ and $a \in K^{*}$ we have

$$
\begin{aligned}
\varphi_{T} & (\Psi(\tau))(v(a)+v F)=\operatorname{sgn}_{T}(a) \operatorname{sgn}_{\Psi(\tau)}(a) \\
& =\operatorname{sgn}_{T}(a) \operatorname{sgn}_{\tau(T)}(a)=\operatorname{sgn}_{T}(a) \operatorname{sgn}_{T}\left(\tau^{-1}(a)\right) \\
& =\operatorname{sgn}_{T}\left(a / \tau^{-1}(a)\right)=\operatorname{sign}\left(\sigma_{K}\left(a / \tau^{-1}(a)\right)\right) \\
& =\Phi(\tau)(v(a)+v F)
\end{aligned}
$$

(since $a / \tau^{-1}(a)$ is a unit and $T$ induces $\sigma_{K}$ ). Thus $\Phi=\varphi_{T} \Psi$ is a bijection. That $\Phi$ is a homomorphism is easily verified; for all $\tau, \rho \in \operatorname{Aut}(K / F)$ and $a \in K^{*}$ we have

$$
\begin{aligned}
& \Phi(\tau \rho)(v(a)+v F)=\operatorname{sign} \sigma_{K}\left(a /(\tau \rho)^{-1}(a)\right) \\
& \quad=\operatorname{sign} \sigma_{K}\left(\left(a / \tau^{-1}(a)\right)\left(\tau^{-1}(a) / \rho^{-1} \tau^{-1}(a)\right)\right) \\
& \quad=\operatorname{sign} \sigma_{K}\left(a / \tau^{-1}(a)\right) \operatorname{sign} \sigma_{K}\left(\tau^{-1}(a) / \rho^{-1} \tau^{-1}(a)\right) \\
& =\Phi(\tau)(v(a)+v F) \Phi(\rho)\left(v\left(\tau^{-1}(a)\right)+v F\right) \\
& =\Phi(\tau)(v(a)+v F) \Phi(\rho)(v(a)+v F) \\
& =(\Phi(\tau) \Phi(\rho))(v(a)+v F) .
\end{aligned}
$$

The next corollary of Theorem 3.2 will be applied in $\S 7$ to the construction of automorphisms.

3.6 THEOREM. Let $\theta: F \rightarrow K$ be a homomorphism such that $K / \theta(F)$ is algebraic, $\sigma_{K} \theta=\sigma_{K}\left|F, v_{K} \theta=v_{K}\right| F$, and $\theta\left(K^{2} \cap F\right)=K^{2} \cap \theta(F)$. Then $\theta$ extends to an automorphism $\theta^{\prime}$ of $K$ with $v_{K} \theta^{\prime}=v_{K}$.

ProOF. Let us adopt the formal point of view that a field extension $K / E$ is a homomorphism $E \rightarrow K$. Let $K / F$ be the extension given by the inclusion map and $L / F$ be the extension given by $\theta$. The covering hypotheses of this section are then satisfied by $K / F$ and $L / F$. Moreover condition 3.2(4) is satisfied (since $\theta$ induces the identity map $v F \rightarrow v(\theta(F))$ and $\left.\theta^{-1}\left(K^{2}\right)=K^{2} \cap F\right)$. Our conclusion follows from Theorem 3.2.

A similar argument yields an analogous theorem for ordered fields. 
3.7 THEOREM. Suppose $S$ and $T$ are orderings of $K$. Let $\theta: F \rightarrow K$ be a homomorphism with $K / \theta(F)$ algebraic, $\theta(F \cap S) \subset T$ and $v_{K} \theta=v_{K} \mid F$. Then $\theta$ extends to an automorphism $\theta^{\prime}$ of $K$ with $\theta^{\prime}(S)=T$.

4. Maximal valued fields. Throughout this section $K$ and $L$ will denote field extensions of a field $F$ which are maximal valued fields with Archimedean real closed residue class fields. Examples of such field extensions include Valente's "completions" at 0-primes [V], and "ultracompletions" of fields either at real Harrison primes $[\mathbf{H W}]$ or at real prime values $[\mathbf{B r} 3, \S 1]$. Note that $K$ and $L$ are real Henselian, and are maximal valued fields with respect to $v_{K}$ and $v_{L}$ (cf., Proposition 2.2).

4.1 THEOREM. Suppose $E$ is a real Henselian extension of $F$. Suppose $S$ and $T$ are orderings of $E$ and $L$, respectively, with $F \cap S=F \cap T$. Suppose $\sigma_{E}(E) \subset \sigma_{L}(L)$ and that there is an injective ordered group homomorphism $\Psi: v E \rightarrow v L$ with $\Psi v_{E}\left|F=v_{L}\right| F$. Then there is an $F$-homomorphism $\Delta: E \rightarrow L$ with $\Delta(S) \subset T$ and with $\Psi v_{E}=v_{L} \Delta$.

We will prove 4.1 at the end of this section after considering some of its corollaries.

4.2 THEOREM. Suppose that $S$ and $T$ are orderings of $K$ and $L$, respectively, with $S \cap F=T \cap F$, that $\sigma_{K}(K)=\sigma_{L}(L)$, and that there exists an isomorphism of ordered groups $\Psi: v K \rightarrow v L$ with $\Psi v_{K}\left|F=v_{L}\right| F$. Then there exists an $F$ isomorphism $\Delta: K \rightarrow L$ with $\Delta(S)=T$ and $v_{L} \Delta=\Psi v_{K}$.

ProOF. Apply Theorem 4.1 with $E=K$. Then the field extension $L / \Delta(K)$ is an immediate extension of a maximal valued field, so $L=\Delta(K)$. That is, $\Delta$ is an isomorphism.

We now apply Theorem 4.2 to obtain an analogue of Theorem 3.2, i.e., an isomorphism theorem for unordered maximal valued fields.

4.3 TheOREM. Suppose that $\sigma_{K}(K)=\sigma_{L}(L)$, that $K^{2} \cap F=L^{2} \cap F$, and that there is an isomorphism of ordered groups $\Psi: v K \rightarrow v L$ with $\Psi v_{K}\left|F=v_{L}\right| F$. Then there exists an $F$-isomorphism $\Delta: K \rightarrow L$ with $v_{L} \Delta=\Psi v_{K}$.

Note that any $F$-isomorphism $\Delta: K \rightarrow L$ induces a map $\Psi_{0}: v K \rightarrow v L$ with $v_{L} \Delta=\Psi_{0} v_{K}$ (Proposition 2.2). In this sense, all the hypotheses of 4.3 for the $F$-isomorphism of $K$ and $L$ are clearly necessary.

PROOF. Let $E_{K}$ and $E_{L}$ denote the (relative) algebraic closures of $F$ in $K$ and $L$, respectively. Then $E_{K}$ and $E_{L}$ are real Henselian algebraic extensions of $F$ (Proposition 2.6). The value group of $E_{K}$ is the set of elements of the value group of $K$ which have some nonzero multiple in the value group of $F$, and similarly for $E_{L}$ (Proposition 2.6). Thus $\Psi$ maps $v E_{K}$ onto $v E_{L}$. Also

$$
E_{K}^{2} \cap F=K^{2} \cap F=L^{2} \cap F=E_{L}^{2} \cap F .
$$

Thus there is an $F$-isomorphism $\Delta_{0}: E_{K} \rightarrow E_{L}$ with $v_{L} \Delta_{0}=\Psi v_{K} \mid E_{K}$ (Theorem 3.2 and Remark 3.3.). Hence there are orderings $S$ and $T$ of $E_{K}$ and $E_{L}$, respectively, with $S \cap F=T \cap F$. By Theorem 4.2 it suffices to show that $S$ extends to an ordering of $K$, and similarly for $T$. We indicate the proof for $S$; the proof for $T$ is identical. $K$ is superpythagorean, so it suffices to show that $-1 \notin S K^{2}[\mathbf{B r} 4$, 
Corollary 8]. But if $-1=a b^{2}$ where $a \in S$ and $b \in K$, then $b \in E_{K}$, so $-1 \in S$, a contradiction.

The next result is a refinement for real Henselian field extensions of Kaplansky's theorem that a maximal valued field with characteristic zero residue class field is a generalized power series field [K, Theorem 6]. It will be applied in Remark 4.6 to the study of the group of automorphisms of $K / F$. Note that the generalized power series field $\bar{K}\left(\left(t^{v K}\right)\right)$ (cf., the last paragraph of $\S 1$ ) has a canonical ordering, call it $P_{K}$, consisting of zero together with all nonzero elements whose term of minimal value has positive coefficient. (Recall that $\bar{K}$ is real closed.)

4.4 THEOREM. Let $P$ be any ordering of $K$. There exists an isomorphism of valued fields $\Delta: K \rightarrow \bar{K}\left(\left(t^{v K}\right)\right)$ with $\Delta(P)=P_{K}$ and $\Delta(F) \subset E\left(\left(t^{v F}\right)\right)$, where $E$ is the algebraic closure of $\bar{F}$ in $\bar{K}$.

ProOF Let $U$ denote the real Henselization of $F$ in $K$ (cf., Proposition 2.8). There exists a homomorphism of valued fields $\Delta_{0}: U \rightarrow E\left(\left(t^{v F}\right)\right)$ with $\Delta_{0}(P \cap U) \subset$ $P_{K}$ (apply Theorem 4.1 with $\mathbf{Q}, U, E\left(\left(t^{v F}\right)\right), P \cap U, P_{K} \cap E\left(\left(t^{v F}\right)\right)$ and the identity map on $v F$ in place of $F, E, L, S, T$, and $\Psi)$. A similar application of Theorem 4.2 says that $\Delta_{0}$ can be lifted to the required isomorphism $\Delta$. (This application of 4.2 requires that we adopt the formal point of view that a field extension $K / F$ is a homomorphism $F \rightarrow K$. In applying 4.2 we let the extension $K / F$ be given by the inclusion map $U \rightarrow K$ and the extension $L / F$ be the composition of $\Delta_{0}$ with the natural map $E\left(\left(t^{v F}\right)\right) \rightarrow \bar{K}\left(\left(t^{v K}\right)\right)$.)

The next result will be applied in $\S 6$ to the study of fixed fields of $K$ under sets of automorphisms.

4.5 THEOREM. Suppose $S$ and $T$ are orderings of $K$ and $\theta: F \rightarrow K$ is a homomorphism with $\theta(F \cap S) \subset T$ and $v_{K} \theta=v_{K} \mid F$. Then $\theta$ extends to an automorphism $\Delta$ of $K$ with $\Delta(S)=T$ and $v_{K} \theta=v_{K}$.

ProOF. As in the proof of Theorem 3.6 we will regard the field extensions $K / F$ and $L / F$ as being given by the inclusion map $F \rightarrow K$ and by $\theta$. Now apply Theorem 4.2 with $\Psi$ the identity map on $v K$.

4.6 REMARK. Let $\operatorname{Aut}_{v}(K / F)$ denote the set of $\tau \in \operatorname{Aut}(K / F)$ with $v_{K} \tau=v_{K}$ and let $\operatorname{Aut}_{v F}(v K)$ denote the set of ordered group automorphisms of $v K$ which fix $v F$. We then have an exact sequence

$$
1 \rightarrow \operatorname{Aut}_{v}(K / F) \rightarrow \operatorname{Aut}(K / F) \rightarrow \operatorname{Aut}_{v F}(v K) \rightarrow 1 .
$$

The exactness at $\operatorname{Aut}_{v F} v K$ can be deduced from either Theorem 4.2 (let $S$ be any ordering of $K$ and $T=S$ ) or Theorem 4.4 (treat $\Delta$ as an identification). Note that if $K / F$ is algebraic, then $\operatorname{Aut}_{v F}(v K)=1$. Arguing as in the proof of Theorem 3.5 (but using 4.5 in place of 3.1 ), we obtain a surjective homomorphism $\Phi: \operatorname{Aut}_{v}(K / F) \rightarrow \operatorname{Hom}\left(v K / v F, \mathbf{Z}^{*}\right)$. When $K / F$ is not algebraic, then $\Phi$ is not necessarily injective, basically because the homomorphisms " $\Delta$ " of Theorem 4.1 are not necessarily unique. It is easy to verify that the kernel of $\Phi$ is exactly the set of those elements of $\operatorname{Aut}_{v}(K / F)$ which are order automorphisms for some (equivalently, for every) ordering of $K$. That there can be many such automorphisms is illustrated by Proposition 7.1 below (consider, for example, the case when $K$ is real closed). 
The remainder of this section will be devoted to the proof of Theorem 4.1.

Let $\theta_{0}: E_{0} \rightarrow L_{0}$ be a maximal extension of the identity map on $F$ to an isomorphism of subfields $E_{0}$ of $E$ and $L_{0}$ of $L$ such that $\theta_{0}\left(S \cap E_{0}\right) \subset T$ and $v_{L} \theta_{0}=\Psi v_{E} \mid E_{0}$. Let $E_{1}$ and $L_{1}$ denote the maximal algebraic extensions of $E_{0}$ and $L_{0}$ in $E$ and $L$, respectively. By Proposition 2.6, $E_{1}$ is real Henselian and, further, $v E_{1} / v E_{0}$ is exactly the torsion part of $v E / v E_{0}$. Similarly, $L_{1}$ is real Henselian and $v L_{1} / v L_{0}$ is the torsion part of $v L / v L_{0}$. Then $\Psi$ carries $v E_{1}$ into $v L_{1}$. Hence by Theorem 3.1, $\theta_{0}$ extends to a homomorphism $\theta_{1}: E_{1} \rightarrow L_{1}$ with $\theta_{1}\left(E_{1} \cap S\right) \subset T$. Now $v_{L} \theta_{1}$ and $\Psi v_{E} \mid E_{1}$ are equivalent extensions of the valuation $v_{L} \theta_{0}=\Psi v_{E} \mid E_{0}$ on $E_{0}$ to the algebraic extension $E_{1}$ (Proposition 2.2). Thus they are equal. Hence by the choice of $\theta_{0}, E_{0}=E_{1}$. Thus $E_{0}$ is algebraically closed in $E$, and so it is real Henselian.

We next claim that $\bar{E}_{0}=\bar{E}$. Just suppose there exists $a \in E$ with $\bar{a} \in \bar{E} \backslash \bar{E}_{0}$. Then $\bar{a}$ is transcendental over $\bar{E}_{0}$ (Proposition 2.6), so $a$ is transcendental over $E_{0}$. We may suppose $a \in S$. By hypothesis there exists $b \in L$ with $\sigma_{L}(b)=\sigma_{E}(a)>0$. Then $b \in T$. Let $\theta_{1}: E_{0}(a) \rightarrow L_{0}(b)$ be the unique extension of $\theta_{0}$ to an isomorphism with $\theta_{1}(a)=b$. Then $v_{L} \theta_{1}=\Psi v_{E} \mid E_{0}(a)$ (e.g., see [Bo, p. 161, Proposition 2]). Now let $c \in E_{0}(a) \cap S$. There exists $d \in E_{0} \cap S$ with $v(c)=v(d)$. By the choice of $b$ we have $\sigma_{E} \mid E_{0}(a)=\sigma_{L} \theta_{1}$ (note that $\sigma_{E} \mid E_{0}=\sigma_{L} \theta_{0}$ by Proposition 2.2). Hence

$$
\sigma_{L}\left(\theta_{1}(c / d)\right)=\sigma_{E}(c / d)>0 .
$$

Thus

$$
\theta_{1}(c)=\theta_{0}(d) \theta_{1}(c / d) \in T \sigma_{L}^{-1}\left(\mathbf{R}^{\cdot 2}\right) \subset T .
$$

Thus $\theta_{1}\left(E_{0}(a) \cap S\right) \subset T$. Thus by the choice of $\theta_{0}$ we must have $E_{0}=E_{0}(a)$, a contradiction. Hence $\bar{E}=\bar{E}_{0}$.

We next argue that $v E=v E_{0}$. Just suppose there exists $a \in E^{*}$ with $v(a) \notin v E_{0}$. We may assume $a \in S$. Then $v(a)+v E_{0}$ is not torsion in $v E / v E_{0}$ (Proposition 2.6), so $a$ is transcendental over $E_{0}$. We can pick $b \in T$ with $v_{L}(b)=\Psi\left(v_{E}(a)\right)$. Let $\theta_{1}: E_{0}(a) \rightarrow L_{0}(b)$ be the unique extension of $\theta_{0}$ with $\theta_{1}(a)=b$. One easily verifies that $v_{L} \theta_{1}=\Psi v_{E} \mid E_{0}(a)\left[\mathbf{B o}\right.$, p. 161, Proposition 1]. Now let $c \in E_{0}(a) \cap S$. There exists a positive integer $n$ and $d \in E_{0} \cap S$ with $v(c)=v\left(a^{n} d\right)$; further, there exists $e \in E_{0}$ with $\sigma_{E}(e)=\sigma_{E}\left(c / a^{n} d\right) \in \mathbf{R}^{\cdot 2}\left(E_{0}(a) / E_{0}\right.$ is totally ramified with $\left.v E_{0}(a)=v E_{0}+\mathbf{Z} v(a)\right)$. Thus $e \in S$. Note that

$$
v_{L}\left(\theta_{1}\left(1-\left(c / a^{n} d e\right)\right)\right)=\Psi v_{E}\left(1-\left(c / a^{n} d e\right)\right)>0
$$

so $\sigma_{L}\left(\theta_{1}\left(c / a^{n} d e\right)\right)=1$. Thus

$$
\theta_{1}(c)=\theta_{1}\left(c / a^{n} d e\right) b^{n} \theta_{0}(d e) \in \sigma_{L}^{-1}(1) T \subset T .
$$

Thus $\theta_{1}\left(E_{0}(a) \cap S\right) \subset T$. Once again we can conclude that $E_{0}=E_{0}(a)$, so $v(a) \in$ $v E_{0}$. This contradiction shows that $v E=v E_{0}$.

It remains to show that $E=E_{0}$. Just suppose that there exists $a \in E \backslash E_{0}$. By the previous paragraphs $E / E_{0}$ is an immediate extension. Thus $a$ is the limit of a pseudoconvergent set $A$ contained in $E_{0}$ which has no limit in $E_{0}$ [K, Theorem 1]. This set is of transcendental type, since otherwise the real Henselian field $E_{0}$ would have a proper algebraic immediate extension [K, Theorem 3], contradicting Proposition 2.10. Since $L$ is a maximal valued field, there is a limit $b$ to the pseudoconvergent set $\theta_{0}(A)$ and hence an extension of $\theta_{0}$ to an isomorphism 
$\theta_{1}: E_{0}(a) \rightarrow L_{0}(b)$ with $\theta_{1}(a)=b$ and $v_{L} \theta_{1}=\Psi v_{E} \mid E_{0}(a)[\mathbf{K}$, Theorem 4 and Theorem 2]. Now let $d \in E_{0}(a) \cap S$. It suffices to show that $\theta_{1}(d) \in T$. Since $E_{0}(a) / E_{0}$ is an immediate extension, there exists $c \in E_{0}$ with $v(c / d-1)>0$. Clearly $c=d(1+c / d-1) \in S$. Then

$$
\theta_{1}(d)=\theta_{0}(c) \theta_{1}(d / c-1+1) \in T \sigma_{L}^{-1}(1) \subset T,
$$

as required.

5. Orderings of higher exact level. Throughout this section $K$ and $L$ denote real Henselian extensions of a field $F$ and $P_{K}, P_{L}$ and $P$ denote "orderings of higher level" (see below) of $K, L$ and $F$, respectively, with

$$
P_{K} \cap F=P=P_{L} \cap F .
$$

In this section we will generalize Theorems 1.2 and 4.2 so as to allow orderings of higher exact level in addition to the classical orderings of the Artin-Schreier theory. As an application we show that generalized real closures at orderings of higher level are determined up to isomorphism by their value groups.

We recall some basic concepts. An ordering of higher level of $F[\mathbf{B}$, p. 866] is a set of the form $H \cup\{0\}$ where $H$ is an additively closed subgroup of the multiplicative group $F^{*}$ such that $F^{*} / H$ is finite cyclic; the exact level of such an ordering of higher level $H \cup\{0\}$ is the order of the group $F^{*} / \mathbf{Z}^{*} H$. Since orderings of higher level appear only in this section, we will refer to them as "Becker orderings" and continue to refer to the classical ordering (i.e., the Becker orderings of exact level one) simply as "orderings."

Each Becker ordering canonically induces a place into $\mathbf{R}$ [B, Theorem 2.2]. By Proposition 2.2, the places induced by $P_{K}$ and $P_{L}$ are $\sigma_{K}$ and $\sigma_{L}$ (cf., 2.3). Condition (1) guarantees that $\sigma_{K}$ and $\sigma_{L}$ have the same restriction to $F$, namely, the place $F \rightarrow \mathbf{R} \cup\{\infty\}$ induced by $P$. We denote this place by $\sigma$ and its associated valuation by $v_{F}$ (or just $v$ ). Since the restrictions of $v_{K}$ and $v_{L}$ to $F$ are equivalent to $v$, we can and do assume without loss of generality that $v_{K}$ and $v_{L}$ restrict to $v$ on $F$.

We begin with a remark describing the "2-primary component" of a Becker ordering, and a technical lemma which will allow us to apply our earlier results on orderings to Becker orderings.

For any Becker ordering $S$, we let $S^{*}=S \backslash\{0\}$.

5.1 REMARK (SEE [BHR, DEFINITION 1.4]). Let $H$ denote the set of elements of $F$ with some odd power in $P$; we call $H$ the 2-primary component of $P$. If $P$ has exact level $2^{m} t$ where $t$ is odd, then $H$ is a Becker ordering of exact level $2^{m}$, $P=H \cap v^{-1}(v(P))$, and $H$ induces $\sigma$.

$H$ can be characterized as the unique Harrison prime of $F$ containing $P$ [HW]; the correspondence $\Delta \mapsto H \cap v^{-1}(\Delta)$ gives a bijection from the set of subgroups $\Delta$ of $\Gamma$ with $\Gamma / \Delta$ cyclic of order $t$ to the set of Becker orderings of $F$ of exact level $2^{m} t$ with 2-primary component $H$.

We will use below only the facts in the first paragraph of 5.1; for the convenience of the reader we give now a proof of these facts avoiding the signature-theoretic language of [BHR]. The proof of the assertions in the second paragraph are left to the interested reader. 
By the basic properties of cyclic groups, $F^{*} / H^{*}$ is cyclic of order $2^{m+1} \cdot \sigma(H) \geq 0$ since if $\sigma(a)<0$ for some $a \in H$, then we would have $a^{t} \in P$ and hence $0>$ $(\sigma(a))^{t}=\sigma\left(a^{t}\right) \geq 0$, a contradiction. Thus $-1 \notin H$. Suppose $a, b \in H$ with $v(a) \geq v(b)$. Then

$$
a+b=b\left(1+a b^{-1}\right) \in H \sigma^{-1}\left(\mathbf{R}^{\cdot 2}\right) \subset H .
$$

This shows $H$ is a Becker ordering of exact level $2^{m}$ inducing $\sigma$. Also if $a \in$ $H \cap v^{-1}(v(P))$, then there exists $c \in P$ with $v(a)=v(c)$, so that

$$
a=c(a / c) \in P \sigma^{-1}\left(\mathbf{R}^{\cdot 2}\right) \subset P .
$$

It follows that $H \cap v^{-1}(v(P))=P$.

For the remainder of this section we let $H_{K}, H_{L}$ and $H$ denote the 2-primary components of $P_{K}, P_{L}$ and $P$, respectively (in the sense of Remark 5.1).

5.2 LEMMA. Suppose $\Psi: v K \rightarrow v L$ is an isomorphism of ordered groups fixing $v F$ such that $\Psi\left(v_{K} P_{K}\right)=v_{L} P_{L}$. Further suppose that either $H_{K}$ is a faithful extension of $H$ (i.e., $K=F H_{K}$ ) or that $H$ is an ordering. Then there exist orderings $S_{K}, S_{L}$ and $S$ of $K, L$, and $F$, respectively, with $S_{K} \cap F=S=S_{L} \cap F$ and $\Psi\left(v\left(S_{K} \cap H_{K}\right)\right)=v\left(S_{L} \cap H_{L}\right)$. If $\theta: K \rightarrow L$ is an $F$-isomorphism with $\theta\left(S_{K}\right)=S_{L}$ and $v_{L} \theta=\Psi v_{K}$, then $\theta\left(P_{K}\right)=P_{L}$.

ProOF. We first prove the existence of $S_{K}, S_{L}$ and $S$. Let $2^{m} t$, where $t$ is odd, denote the exact level of $P_{K}$. First note that $H_{K} \cap F=H=H_{L} \cap F$. Further, $\Psi\left(v H_{K}\right)=v H_{L}$ since $v H_{K} / v P_{K}$ is exactly the set of elements of $v K / v P_{K}$ of odd order, and similarly for $L$. (After all, suppose $a \in K$ has $n v(a)=v(b)$ for some odd integer $n$ and $b \in P_{K}$. Then possibly replacing $a$ by $-a$, we have $a^{n}=b\left(a^{n} / b\right) \in P_{K} \sigma_{K}^{-1}\left(\mathbf{R}^{\cdot 2}\right) \subset P_{K}$, so $a \in H_{K}$.) We may assume without loss of generality that $H_{K}$ is not an ordering, since otherwise so are $H_{L}$ and $H$ (because then $\left.v H_{L}=\Psi\left(v H_{K}\right)=\Psi(v K)=v L\right)$, and so we could just set $S_{K}=H_{K}, S_{L}=H_{L}$ and $S=H$.

Now assume $H$ is an ordering. Then let $S_{K}$ be any ordering of $K . v\left(S_{K} \cap H_{K}\right)$ has index $2^{m+1}$ in $v K$ (apply [Br5, Theorem 2.1], noting that the exact level of $H_{K}$ in the sense of $[\mathbf{B r 5}]$ is $m+1$, not $\left.2^{m}\right)$. Since $\Psi\left(v H_{K}\right)=v H_{L}$, then $H_{L}$ has exact level $2^{m}$ and $v H_{L} \supset \Psi\left(v\left(S_{K} \cap H_{K}\right)\right)$. Thus there exists an ordering $S_{L}$ of $L$ with $v\left(S_{L} \cap H_{L}\right)=\Psi\left(v\left(S_{K} \cap H_{K}\right)\right)[\mathbf{B r 5}$, Lemma 6.3A]. Then

$$
\begin{aligned}
v\left(H \cap\left(F \cap S_{L}\right)\right) & =v\left(H_{L} \cap S_{L}\right) \cap v F \\
& =v\left(S_{K} \cap H_{K}\right) \cap v F=v\left(H \cap\left(F \cap S_{K}\right)\right) .
\end{aligned}
$$

Hence $F \cap S_{L}=F \cap S_{K}\left[\mathbf{B r} \mathbf{5}\right.$, Theorem 1.1]. We can then set $S=F \cap S_{L}$.

Next suppose that $H$ is not an ordering and $H_{K} / H$ is a faithful extension of $H$. Then $H_{L}$ is also faithful over $H$ (since $\left.\Psi\left(v H_{K}\right)=v H_{L}\right)$. There exist distinct orderings $T_{K}$ and $S_{K}$ of $K$ with $v\left(S_{K} \cap H_{K}\right)=v\left(S_{K} \cap T_{K} \cap H_{K}\right)$ and with $v K / v\left(S_{K} \cap H_{K}\right)$ cyclic of order $2^{m+1}$ [Br5, Theorem 2.1 and formula (2) of $\left.\S 5\right]$. Since $\Psi(v K)=v L$ and $\Psi\left(v\left(S_{K} \cap H_{K}\right)\right) \subset \Psi\left(v H_{K}\right)=v H_{L}$, there exist distinct orderings $T_{L}$ and $S_{L}$ of $L$ with

$$
v\left(T_{L} \cap S_{L} \cap H_{L}\right)=\Psi\left(v\left(T_{K} \cap S_{K} \cap H_{K}\right)\right)
$$


[Br5, Lemma 6.3A]. Then

$$
\begin{aligned}
v\left(S_{L} \cap T_{L} \cap H\right) & =v(F) \cap v\left(S_{L} \cap T_{L} \cap H_{L}\right) \\
& =v(F) \cap v\left(S_{K} \cap T_{K} \cap H_{K}\right)=v\left(S_{K} \cap T_{K} \cap H\right) .
\end{aligned}
$$

Therefore the natural map

$$
v F / v\left(S_{L} \cap T_{L} \cap H\right) \rightarrow v L / v\left(S_{L} \cap T_{L} \cap H_{L}\right)
$$

is injective. Hence $v F / v\left(S_{L} \cap T_{L} \cap H\right)$ is cyclic. Since $v H$ and $v\left(S_{L} \cap T_{L} \cap F\right)$ both contain $v\left(S_{L} \cap T_{L} \cap H\right)$, they must be comparable. But $v\left(S_{L} \cap T_{L} \cap F\right)$ has index at most 2 in $v F$, and $H$ is not an ordering, so $v\left(S_{L} \cap T_{L} \cap F\right) \supset v H$. Thus $v F / v\left(S_{L} \cap T_{L} \cap H\right)$ has order $\epsilon$ xactly $2^{m+1}$ [Br5, Theorem 2.1 and $\S 5$, formula (2)]. Thus the map (4) is surjective. This implies that the natural map $v F / v\left(S_{L} \cap T_{L} \cap F\right) \rightarrow v L / v\left(S_{L} \cap T_{L}\right)$ is surjective. Hence

$$
v F \neq v\left(S_{L} \cap T_{L} \cap F\right) .
$$

Therefore $S_{L} \cap F \neq T_{L} \cap F$. A similar argument shows $S_{K} \cap F \neq T_{K} \cap F$. Consequently $S_{K} \cap T_{K} \cap F=S_{L} \cap T_{L} \cap F$ (use (3) to apply [Br5, Lemma 6.3A]). Thus the ordering $S_{K} \cap F$ of $F$ equals either $S_{L} \cap F$ or $T_{L} \cap F$. Without loss of generality it equals $S_{L} \cap F$. If we let $S=S_{L} \cap F$, we have $S_{K} \supset S, S_{L} \supset S$ and, by (2) $\Psi\left(v\left(S_{K} \cap H_{K}\right)\right)=v\left(S_{L} \cap H_{L}\right)$. This completes the proof of the existence of orderings $S_{K}, S_{L}$ and $S$ of $K, L$ and $F$ with $S_{K} \cap F=S=S_{L} \cap F$ and $\Psi\left(v\left(S_{K} \cap H_{K}\right)\right)=v\left(S_{L} \cap H_{L}\right)$. Now suppose $S_{K}, S_{L}$ and $S$ are such orderings and $\theta: K \rightarrow L$ is an $F$-isomorphism with $\theta\left(S_{K}\right)=S_{L}$ and $v_{L} \theta=\Psi v_{K}$. We first claim that $\theta\left(H_{K}\right)=H_{L}$. This is clear if $v\left(S_{K} \cap H_{K}\right)=v K$ since then $S_{K}=H_{K}$ (and, similarly, $\left.S_{L}=H_{L}\right)\left[\right.$ Br5, Theorem 2.1]. Now suppose $v\left(S_{K} \cap H_{K}\right) \neq v K$. Then

$$
H_{K}=\left(S_{K} \cap v^{-1}(\Delta)\right) \cup\left(\left(-S_{K}\right) \cap v^{-1}\left(\Delta^{\prime} \backslash \Delta\right)\right) \cup\{0\}
$$

where $\Delta=v\left(S_{K} \cap H_{K}\right)$ and $\Delta^{\prime}=2^{m} v K+\Delta[\mathbf{B r 5}$, Remark 2.3A]. We have a similar formula for $H_{L}$. Since $\theta$ induces $\Psi$ (which maps $\Delta$ to $v\left(S_{L} \cap H_{L}\right.$ ) and similarly for $\left.\Delta^{\prime}\right)$, we deduce that $\theta\left(H_{K}\right)=H_{L}$ in all cases. Hence by Remark 5.1, $\theta\left(P_{K}\right)=\theta\left(H_{K} \cap v^{-1}\left(v P_{K}\right)\right)=H_{L} \cap v^{-1}\left(v P_{L}\right)=P_{L}$. Lemma 5.2 is proved.

We now generalize part of Theorem 1.2; uniqueness will be handled in Theorem 5.5 below.

5.3 ThEOREM. Suppose $K$ and $L$ are algebraic extensions of $F$. Suppose $v K=$ $v L$ and $v P_{K}=v P_{L}$. Further suppose that either $P$ has odd level or that $P_{K}$ is a faithful extension of $P$. Then there exists an $F$-isomorphism $\Phi: K \rightarrow L$ with $\Phi\left(P_{K}\right)=P_{L}$.

As in $\S 1$, we are implicitly assuming in the statement of 5.3 that $v K$ and $v L$ have been canonically identified with subgroups of a divisible hull of $v F$.

PrOOF. Take $\Psi$ in Lemma 5.2 to be the identity map. Now apply Theorem 1.2 and Lemma 5.2. Note that $P$ has odd exact level if and only if $H$ is an ordering, and that if $P_{K}$ is a faithful extension of $P$ (i.e., $K=F P_{K}$ ), then surely $H_{K}$ is a faithful extension of $H$ (i.e., $K=F H_{K}$ ).

The conditions in Theorem 5.3 that $v K=v L$ and $v P_{K}=v P_{L}$ are clearly necessary. We return to Example 1.1 in order to illustrate that we cannot drop from Theorem 5.3 the hypothesis that either $P$ have odd exact level or $F P_{K}=K$. (Of course, we can replace $F P_{K}=K$ by $F H_{K}=K$.) 
5.4 EXAMPLE (SEE [BHR, ExAmPle 3.17]). Let $K, L$ and $F$ be as in Example 1.1. Then $K$ and $L$ are not $F$-isomorphic even though $\bar{K}$ and $\bar{L}$ are $\bar{F}$-isomorphic, $v K=v L$, and every Becker ordering $P$ of $F$ extends to a Becker ordering $P_{K}$ of $K$ and a Becker ordering $P_{L}$ of $L$. Indeed if $P$ is a Becker ordering of $F$ of even exact level, then $v P_{K}=v P_{L}$ but $P_{K}$ is not a faithful extension of $P$ (i.e., $K \neq F P_{K}$ ). Also if $P$ is a Becker ordering of $F$ of odd exact level, then $v P_{K} \neq v P_{L}$. (These assertions are easily checked using Remark 5.1 and the analysis of Becker orderings of 2-power level in [Br5, Br6].)

5.5 THEOREM. Suppose $K$ and $L$ are algebraic extensions of $F$ and there exists an $F$-isomorphism from $K$ to $L$ mapping $P_{K}$ onto $P_{L}$. If $H_{K}$ is a faithful extension of $H$, there is exactly one such isomorphism; otherwise, there are exactly two.

To say that $H_{K}$ extends $H$ faithfully is just to say that any power of two dividing the exact level of $P_{K}$ also divides the exact level of $P$.

PROOF. First suppose $H_{K}$ is an ordering. Then $H_{K}$ is a faithful extension of $H$. Moreover an $F$-isomorphism from $K$ to $L$ mapping $P_{K}$ to $P_{L}$ clearly maps $H_{K}$ to $H_{L}$ and hence is uniquely determined (Theorem 3.1). Next suppose $H_{K}$ is not an ordering. Then there exists a chain of orderings $\left(H_{i}\right)_{i \geq 0}$ of $K$ with $H_{m}=H_{K}$ for some $m \geq 2[\mathbf{H}, \mathbf{B r 6}]$. Let $\Delta=v\left(H_{0} \cap H_{1} \cap H_{m}\right) ; \bar{\Delta}$ is a subgroup of $v H_{K}$ such that $v K / \Delta$ is cyclic of order $2^{m}$ [Br6, §2, Claim 1; Br5, §5, formula (2)]. Our hypotheses guarantee that $v K=v L, v P_{K}=v P_{L}$, and so $v H_{K}=v H_{L}$. Hence there exists a unique set $\left\{H_{0}^{\prime}, H_{1}^{\prime}\right\}$ of two distinct orderings of $L$ with $v\left(H_{0}^{\prime} \cap H_{1}^{\prime} \cap H_{L}\right)=\Delta$ [Br5, Lemma 6.3A]. Thus there exist Becker orderings $H_{i}^{\prime}, i \geq 2$, such that $\left(H_{i}^{\prime}\right)_{i \geq 0}$ is a chain of orderings of $L$ with $H_{m}^{\prime}=H_{L}$ [Br5, Corollary 6.5].

Now let $\Phi: K \rightarrow L$ be any $F$-isomorphism with $\Phi\left(P_{K}\right)=P_{L}$. Then $\Phi\left(H_{K}\right)=$ $H_{L}$, so

$$
\begin{gathered}
v\left(\Phi\left(H_{0}\right) \cap \Phi\left(H_{1}\right) \cap H_{L}\right)=v\left(\Phi\left(H_{0} \cap H_{1} \cap H_{K}\right)\right) \\
=v\left(H_{0} \cap H_{1} \cap H_{K}\right)=v\left(H_{0}^{\prime} \cap H_{1}^{\prime} \cap H_{L}\right) .
\end{gathered}
$$

Thus $\Phi\left(\left\{H_{0}, H_{1}\right\}\right)=\left\{H_{0}^{\prime}, H_{1}^{\prime}\right\}\left[\mathbf{B r 5}\right.$, Lemma 6.3A]. Hence $\Phi\left(H_{0}\right)$ is either $H_{0}^{\prime}$ or $H_{1}^{\prime}$. Thus by Theorem 3.1, there are at most two $F$-isomorphisms from $K$ to $L$ mapping $P_{K}$ onto $P_{L}$.

Next observe that $\left\{H_{0} \cap F, H_{1} \cap F\right\}=\left\{H_{0}^{\prime} \cap F, H_{1}^{\prime} \cap F\right\}$. Suppose that $H_{K}$ is a faithful extension of $H$. Then so is $H_{L}$ (the equality $v H_{K}=v H_{L}$ says $H_{K}$ and $H_{L}$ have the same exact level). Hence $\left(H_{i}^{\prime} \cap F\right)_{i \geq 0}$ is a chain of orderings [Br6, Lemma 3.2]. Thus $H_{0}^{\prime} \cap F \neq H_{1}^{\prime} \cap F$. Hence $H_{0} \cap F$ must be either $H_{0}^{\prime} \cap F$ or $H_{1}^{\prime} \cap F$ but not both. If $H_{0} \cap F=H_{0}^{\prime} \cap F$, then $H_{0} \cap F \neq H_{1}^{\prime} \cap F$, so $\Phi\left(H_{0}\right) \neq H_{1}^{\prime}$, so $\Phi\left(H_{0}\right)$ must be $H_{0}^{\prime}$. By Theorem 3.1, this uniquely determines $\Phi$. Similarly, if $H_{0} \cap F=H_{1}^{\prime} \cap F$, then $\Phi$ is uniquely determined by the condition that $\Phi\left(H_{0}\right)$ must be $H_{1}^{\prime}$. In either case, there is only one such isomorphism $\Phi$.

Finally suppose $H_{K}$ is not a faithful extension of $H$. Then $H_{L}$ is not a faithful extension of $H$ and $\left(H_{i} \cap F\right)_{i \geq 0}$ is not a chain of orderings of $F$. Thus [Br6, Lemma $3.2]$

$$
H_{0} \cap F=H_{1} \cap F=H_{0}^{\prime} \cap F=H_{1}^{\prime} \cap F .
$$

Then $H_{0}^{\prime}$ and $H_{1}^{\prime}$ are extensions of $H_{0} \cap F$ to orderings of $L$. Hence there exist $F$-isomorphisms $\Phi_{i}: K \rightarrow L$ with $\Phi_{i}\left(H_{0}\right)=H_{i}^{\prime}, i=0,1$. Then by Lemma 5.2 (with $\Psi$ the identity map and $S_{K}=H_{0}$ and $S_{L}=H_{i}^{\prime}$ ) we have $\Phi_{i}\left(P_{K}\right)=P_{L}$ for 
$i=0,1$. (To show that $v\left(H_{0} \cap H_{K}\right)=v\left(H_{i}^{\prime} \cap H_{L}\right)$ use the choice of $H_{0}, H_{1}, H_{0}^{\prime}$ and $H_{i}^{\prime}$ and $[\mathbf{B r 5}, \S 5$, formula (2)].) Thus there are at least two (and hence exactly two) $F$-isomorphisms from $K$ to $L$ mapping $P_{K}$ to $P_{L}$.

A real closure of $(F, P)$ is a pair $(E, T)$ where $E$ is a maximal algebraic extension of $F$ admitting a faithful extension of $P$ and $T$ is just such an extension of $P$ to $E$ $[B, \S 4]$.

5.6 TheOREM. Suppose $\left(K, P_{K}\right)$ and $\left(L, P_{L}\right)$ are real closures of $(F, P)$. There exists an $F$-isomorphism from $K$ onto $L$ if and only if $v K=v L$. If such an isomorphism exists, it is unique and maps $P_{K}$ onto $P_{L}$.

We should note that the hypotheses of Theorem 5.6 imply the covering hypotheses for this section [B, Theorem 5.1]. We are implicitly assuming, moreover, that as earlier we have identified the restrictions of $v_{K}$ and $v_{L}$ to $F$ and identified $v K$ and $v L$ with subgroups of the divisible hull of $v F$.

PrOOF OF 5.6. The necessity of the condition $v K=v L$ is obvious. Now suppose $v K=v L$. By Theorems 5.3 and 5.5 it suffices to show that $v P_{K}=v P_{L}$. Let $n$ denote the exact level of $P$. Then $v K / v P_{K}$ is cyclic of order $n$, so $v P_{K} \supset n v K$ (consider the exact sequence

$$
1 \rightarrow \mathbf{R}^{*} / \mathbf{R}^{\cdot 2} \rightarrow K^{*} / P_{K}^{\cdot} \rightarrow v K / v P_{K} \rightarrow 0
$$

induced by the inclusion map $\sigma_{K}^{-1}\left(\mathbf{R}^{*}\right) \rightarrow K^{*}$ and the valuation $v_{K}$ ). Let $p$ be any prime dividing $n$, say with $n=p^{t} m$ where $p \nmid m$. The $p$-primary components of $v K / n v K$ and $v K / v P_{K}$ are both cyclic of order $p^{t}$ [B, Theorem 4.1(ii)] and hence correspond bijectively under the natural surjection

$$
v K / n v K \rightarrow v K / v P_{K} .
$$

Since this is true for all $p$, the map (5) is a bijection. Thus $n v K=v P_{K}$. Similarly, $v P_{L}=n v L$. Thus

$$
v P_{K}=n v K=n v L=v P_{L}
$$

as required.

Theorem 4.2 can be generalized in the same way that Theorem 1.2 was.

5.7 THEOREM. Suppose $K$ and $L$ are maximal valued fields with Archimedean real closed residue class fields. Suppose $\sigma_{K}(K)=\sigma_{L}(L)$ and that there exists an isomorphism of ordered groups $\Psi: v K \rightarrow v L$ with $\Psi v_{K}\left|F=v_{L}\right| F$ and $\Psi\left(v P_{K}\right)=$ $v P_{L}$. Suppose further that either $P_{K}$ is a faithful extension of $P$ or $P$ has odd exact level. Then there exists an $F$-isomorphism $\Delta: K \rightarrow L$ with $\Delta\left(P_{K}\right)=P_{L}$. $T)$.

ProOF. Apply Lemma 5.2 and Theorem 4.2 (with $S_{K}$ and $S_{L}$ in place of $S$ and

6. Fixed subfields of real Henselian fields. Throughout this section $K$ will denote a real Henselian field and $K / F$ a field extension. We study here the fixed subfields of $K$, i.e., the fixed fields $K^{H}$ of groups $H$ of automorphisms of $K$. We regard $K$ as having the interval topology for any of its orderings. (These topologies all coincide. If $K$ has more than one ordering, then $v_{K}$ is nontrivial and all these topologies equal its valuation topology.) 
6.1 Proposition. Suppose $F$ is a fixed subfield of $K$. Then $F$ is topologically closed in $K$ and the algebraic closure of $F$ in $K$ is a multiquadratic extension of $F$.

Recall that an extension $E / F$ is multiquadratic if $\operatorname{Aut}(E / F)$ has fixed field $F$ and exponent 2. The proposition implies the well-known fact that any Archimedean real closed field (e.g., R) has no nontrivial automorphisms. (Any such field has no closed proper subfields.)

PrOOF OF 6.1. Let $E$ denote the algebraic closure of $F$ in $K$. If $\tau \in \operatorname{Aut}(K / F)$, then $\tau(E)=E$, so that

$$
F \subset E^{\mathrm{Aut}(E / F)} \subset K^{\mathrm{Aut}(K / F)}=F .
$$

Thus $F=E^{\operatorname{Aut}(E / F)}$, i.e., $E / F$ is Galois. $E$ is real Henselian by Proposition 2.6. $\operatorname{Aut}(E / F)$ has exponent two by Theorem $3.5 \mathrm{~A}$. Thus $E / F$ is a multiquadratic extension. That $F$ is topologically closed in $K$ follows from the fact that all automorphisms of $K$ are continuous (Proposition 2.2).

6.2 COROLLARY. If $K$ is a real closed field and $F$ is a fixed subfield, then $F$ is algebraically and topologically closed in $K$. In particular, $F$ is real closed.

The above corollary implies, for example, the well-known fact that if $K$ is a real closure of a subfield $F$, then $K$ has no nontrivial $F$-automorphisms (the fixed field of any such automorphism must be algebraically closed in $K$, i.e., be all of $K$ ).

PROOF OF 6.2. Let $E$ be the algebraic closure of $F$ in $K$. By $6.1, F$ is topologically closed in $K$ and $E / F$ is multiquadratic. But $E$ is real closed (Lemma 2.4 ) and so is not quadratic over any subfield. Thus $E=F$, as required.

The Laurent series field $\mathbf{R}((x))$ is clearly a fixed subfield of its quadratic extension $\mathbf{R}((x))\left[x^{1 / 2}\right]$. This illustrates that we cannot drop the hypothesis that $K$ is real closed in Corollary 6.2. In fact in $\S 8$ we will give an example of a fixed subfield of a real Henselian field $E$ which is not algebraically closed in $E$ and whose algebraic closure in $E$ is not itself a fixed subfield of $E$. Also, in both $\S 7$ and in $\S 8$ we will construct examples which show that the converse of Proposition 6.1 does not hold in general. Our next theorem describes a situation in which this converse does hold.

6.3 THEOREM. Suppose that $K$ is a maximal valued field with Archimedean real closed residue class field. Let $F$ be a subfield of $K$ which is topologically closed in $K$ and whose algebraic closure in $K$ is a multiquadratic extension of $F$. Then $F$ is a fixed subfield of $K$.

6.4 EXAMPLES. (A) Let $F$ be a real closed subfield of a real closed field $K$. Then $F$ and $F((x))$ are fixed subfields of the field of Laurent series $K((x))$. (Actually it is easy to check directly that $F$ is a fixed subfield of $F((x))$ for any field $F$ of characteristic zero.)

(B) Let $R$ be the field of real algebraic numbers (i.e., the algebraic closure of $\mathbf{Q}$ in $\mathbf{R})$. Some of the fixed subfields of $\mathbf{R}((x))$ are $\mathbf{R}, \mathbf{R}\left(\left(x^{2}\right)\right), R, R((x))$ and $R\left(\left(x^{2}\right)\right)$. (Note that the canonical topology on $\mathbf{R}((x))$ is the $x$-adic topology, and $\mathbf{R}$ and $R$ are discrete in this topology).

(C) Any field which is a maximal field with respect to a nontrivial valuation having Archimedean real closed residue class field has a fixed subfield isomorphic to $R$.

Before proving 6.3 we combine it with Proposition 6.2 to obtain the following. 
6.5 COROLlary. Suppose $K$ is a real closed field and $\left(K, v_{K}\right)$ is a maximal valued field. The fixed subfields of $K$ are exactly the subfields of $K$ which are algebraically and topologically closed in $K$.

We devote the remainder of this section to the proof of Theorem 6.3.

Let $\alpha \in K \backslash F$. We must show $\alpha$ is moved (i.e., is not mapped to itself) by some $F$-automorphism of $K$. Clearly, it suffices to show that some element of $F(\alpha)$ is moved by such an automorphism. Let us fix an ordering $P$ of $K$. By Theorem 4.5 it suffices to prove the following.

ClaIM. There exists an ordering $T$ of $K$, a subfield $L$ of $K$ containing $F$, and an $F$-homomorphism $\theta: L \rightarrow K$ such that $\theta(P \cap L) \subset T$ and $v \theta=v \mid L$ and such that $\theta$ moves some element of $F(\alpha) \cap L$.

The proof of this claim will require the consideration of a number of cases. We let $E$ denote the algebraic closure of $F$ in $K$. Note that $E$ and $F$ are real Henselian (Propositions 2.6 and 2.9, respectively).

First suppose $\alpha \in E$. Since $E / F$ is multiquadratic, there exists an $F$-automorphism $\theta$ of $E$ moving $\alpha$. Since $F$ is a Henselian valued field and $E / F$ is algebraic, then $v \theta=v \mid E$. To prove the Claim in this case (with $L=E$ ) it suffices to show that the ordering $\theta(P \cap E)$ of $E$ extends to an ordering of $K$. Since $E$ is algebraically closed in $K$, this can be proved by the argument (showing that $S$ extends to $K$ ) at the end of the proof of Theorem 4.3.

Henceforth we may assume $\alpha \notin E$, i.e., $\alpha$ is transcendental over $F$.

Next suppose $F(\alpha) / F$ is an immediate extension (i.e., that $v(F(\alpha))=v F$ and $\overline{F(\alpha)}=\bar{F})$. Then $\alpha$ is the limit of a pseudoconvergent set $A$ in $F$ which has no limit in $F[\mathbf{K}$, Theorem 1]. This pseudoconvergent set cannot be of algebraic type since otherwise the real Henselian field $F$ would have an immediate proper algebraic extension [K, Theorem 3], contradicting Proposition 2.10. Also note that $A$ has a nontrivial breadth [K, p. 304]; otherwise $\alpha$ is in $F$ since $F$ is topologically closed in $K$. Pick $b \in K^{\circ}$ in the breadth of $A$. Then there exists an $F$-isomorphism of valued fields $\theta: F(\alpha) \rightarrow F(\alpha+b)$ with $\theta(\alpha)=\alpha+b[\mathbf{K}$, Lemma 3 and Theorem 2]. It remains to show that $\theta(P \cap F(\alpha)) \subset P$ (take $L=F(\alpha)$ and $T=P$ in the Claim). Suppose $a \in P \cap F(\alpha)$. Since. $F(\alpha) / F$ is immediate there exists $c \in F$ with $v(a / c-1)>0$, so $v(\theta(a) / c-1)>0$. Thus $\theta(a)=a(c / a)(\theta(a) / c) \in P \sigma_{K}^{-1}(1) \subset P$, as required. Thus the Claim holds when $F(\alpha) / F$ is immediate.

We will therefore assume for the remainder of the proof that $F(\alpha) / F$ is not immediate.

Suppose now that $\bar{F} \neq \overline{F(\alpha)}$. Then we can find a unit $\beta \in F(\alpha)$ with $\bar{\beta} \notin \bar{F}$; without loss of generality, $\sigma_{K}(\beta)>0 . \bar{\beta}$ is transcendental over $\bar{F}$ since $\bar{F}$ is real closed. Note that $v K \neq 0$ (otherwise $K$ is Archimedean real closed, so $F=K$, contradicting that $\alpha \notin F)$. Pick $b \in K^{*}$ with $v(b)>0$. There exists an $F$ isomorphism of valued fields $\theta: F(\beta) \rightarrow F(\beta+b)$ with $\theta(\beta)=\beta+b$ (note $\beta+b$ is transcendental over $F$ since $\overline{\beta+b}=\bar{\beta}$ is transcendental over $\bar{F}$ ) [Bo, p. 161, Proposition 2]. It remains in this case to show that $\theta(F(\beta) \cap P) \subset P$. If $a \in P \cap F(\beta)$, then since $F(\beta) / F$ is unramified, there exists $c \in F \cap P$ with $v(c)=v(a)$. But then $\theta(a)=c \theta(a / c) \in c \theta\left(\sigma_{K}^{-1}\left(\mathbf{R}^{\cdot 2}\right)\right)$ which is contained in $P$ since $\theta$ induces the identity map on $\overline{F(\beta)}=\bar{F}(\bar{\beta})=\overline{F(\beta+b)}$. 
We can henceforth assume that the field extension $F(\alpha) / F$ is totally ramified (i.e., $\bar{F}=\overline{F(\alpha)}$ ) as well as being transcendental and not immediate. Thus $v(F(\alpha)) / v F$ must be nontrivial; let us suppose for the moment that it is not torsion. Then there exists $\beta \in F(\alpha)$ with no nonzero multiple of $v(\beta)$ in $v F$. Without loss of generality, $\beta \in P$ and $v(\beta)<0$. There exists an $F$-isomorphism of valued fields $\theta: F(\beta) \rightarrow F(\beta+1)$ with $\theta(\beta)=\beta+1$. Once again we have $\theta(P \cap F(\beta)) \subset P$. To show this it suffices to show that $\theta(P \cap F[\beta]) \subset P$. Suppose $a=\sum a_{i} \beta^{i} \in P \cap F[\beta]$ (where the coefficients $a_{i}$ are in $F$ ). Let $a_{j} \beta^{j}$ be the term of $a$ of smallest value; then $a_{j} \in P$. The term of

$$
\theta(a)=\sum a_{i}(\beta+1)^{i}
$$

of smallest value is $a_{j}(\beta+1)^{j}$. Since this term is obviously in $P$, so is $\theta(a)$. After all,

$$
\theta(a)=a_{j}(\beta+1)^{j}\left(\theta(a) / a_{j}(\beta+1)^{j}\right) \in P \sigma_{K}^{-1}(1) \subset P .
$$

Hence we may assume that $v(F(\alpha)) / v F$ is torsion.

Recall that by our previous work we are now assuming without loss of generality that $F(\alpha) / F$ is transcendental and totally ramified and that $v(F(\alpha)) / v F$ is nontrivial and torsion. First note $E(\alpha) / F(\alpha))$ is algebraic, so that $\overline{E(\alpha)} / \overline{F(\alpha)}$ is algebraic and $v(E(\alpha)) / v(F(\alpha))$ is torsion. From the first fact we deduce that $\overline{E(\alpha)}=\bar{E}$ (both equal the real closed field $\bar{F}=\overline{F(\alpha)}$ ). From the second we deduce that $v(E(\alpha)) / v F$, and hence $v(E(\alpha)) / v E$, are torsion. However, $v K / v E$ is torsionfree (Proposition 2.6). Hence $v(E(\alpha))=v E$. That is, $E(\alpha) / E$ is an immediate extension.

Since $E / F$ is multiquadratic, $v E / v F$ is a group of exponent 2. Hence $v(F(\alpha)) / v F$ is a nontrivial group of exponent two (since $v(F(\alpha)) \subset v(E(\alpha)) \subset v E$ ). Thus there exists $\beta \in F(\alpha)$ with $v(\beta) \notin v F$ but $2 v(\beta) \in v F$. Note that $\beta \notin E$ since $E \cap F(\alpha)=F$. Hence there exists $a \in F$ with $v\left(\beta^{2} a\right)=0$; we may even assume $\sigma_{K}\left(\beta^{2} a\right)=1$ (since $\bar{F}=\overline{F(\alpha)}$ ). By Hensel's Lemma we conclude that $a=c^{2}$ for some $c \in K$; note that $c \in E$ by the choice of $E$. Since

$$
0<v\left(\beta^{2} c^{2}-1\right)=v(\beta c-1)+v(\beta c+1),
$$

we may assume $v(\beta c-1)>0$ (otherwise replace $c$ by $-c$ ). Note that $c \notin F$ since $-v(c)=v(\beta) \notin v F$. Thus $F[c] / F$ is a quadratic extension. Thus there exists an $F$-isomorphism $\theta$ of $E$ with $\theta(c)=-c$. Since $E(\alpha) / E$ is immediate, $\beta$ is the limit of some pseudo convergent set $\left(b_{\lambda}\right)_{\lambda \in \Lambda}$ in $E$ which has no limit in $E$. Since $E$ is real Henselian, $v \theta=v \mid E$ (i.e., $\theta$ is an isomorphism of valued fields, cf., Proposition 2.2). Thus $\left(\theta\left(b_{\lambda}\right)\right)_{\lambda \in \Lambda}$ must be a pseudoconvergent set; since $K$ is a maximal valued field it must have a limit, say $\beta^{\prime}$, in $K[\mathbf{K}$, Theorem 4]. The pseudo convergent sets $\left(b_{\lambda}\right)_{\lambda \in \Lambda}$ and $\left(\theta\left(b_{\lambda}\right)\right)_{\lambda \in \Lambda}$ must be of transcendental type, since if $\left(b_{\lambda}\right)_{\lambda \in \Lambda}$ were of algebraic type, then the Henselian valued field $(E, v \mid E)$ would admit a proper immediate algebraic extension [K, Theorem 3], contradicting Proposition 2.10. Hence there exists an extension of $\theta$ to an isomorphism of valued fields $\theta^{\prime}: E(\beta) \rightarrow E\left(\beta^{\prime}\right)$ with $\theta^{\prime}(\beta)=\beta^{\prime}\left[\mathbf{K}\right.$, Theorem 2]. Now $v\left(\beta-c^{-1}\right)>v(\beta)$, so (applying $\left.\theta^{\prime}\right) v\left(\beta^{\prime}+c^{-1}\right)>v(\beta)$. Also $v\left(c^{-1}-\theta^{\prime}\left(c^{-1}\right)\right)=v\left(2 c^{-1}\right)=v(\beta)$. Thus

$$
v\left(\beta-\beta^{\prime}\right)=v\left(\beta-c^{-1}+c^{-1}-\theta\left(c^{-1}\right)-\left(c^{-1}+\beta^{\prime}\right)\right)=v(\beta) .
$$

Thus $\beta \neq \beta^{\prime}$, i.e., $\theta^{\prime}$ moves $\beta$. In order to prove the Claim (with $L=E(\beta)$ ) it remains only to show that $\theta^{\prime}(P \cap E(\beta)) \subset T$ for some ordering $T$ of $K$. If 
$\theta^{\prime}(P \cap E(\beta)) \subset P$ we are done. Otherwise $v\left(\theta^{\prime}(P \cap E(\beta)) \cap P\right)$ has index 2 in $v\left(E\left(\beta^{\prime}\right)\right)=v E[\operatorname{Br} \mathbf{1}$, Proposition]. But $v K / v E$ is torsion-free. Thus $v\left(\theta^{\prime}(P \cap E(\beta)) \cap P\right)$ is contained in a subgroup $\Delta$ of $v(K)$ of index two not containing $v E$. There exists an ordering $T$ of $K$ with $v(T \cap P)=\Delta[\operatorname{Br} 1]$. Then since $v E=v E(\beta)=v E\left(\beta^{\prime}\right)$,

$$
v E \cap \Delta \supset v\left(P \cap \theta^{\prime}(P \cap E(\beta))\right)
$$

equality follows from this inclusion since both groups have index two in $v E$. Thus we have

$$
\begin{aligned}
v\left(T \cap P \cap E\left(\beta^{\prime}\right)\right) & =v\left(E\left(\beta^{\prime}\right)\right) \cap v(T \cap P) \\
& =v E \cap \Delta=v\left(\theta^{\prime}(P \cap E(\beta)) \cap\left(P \cap E\left(\beta^{\prime}\right)\right)\right) .
\end{aligned}
$$

Thus

$$
T \supset T \cap E\left(\beta^{\prime}\right)=\theta^{\prime}(P \cap E(\beta))
$$

[Br1, Proposition]. This completes the proof of the Claim, and hence of Theorem 6.3 .

7. Extensions of transcendence degree one. In this section we will present an example of a real Henselian transcendence degree one extension $K$ of a field $F$ such that $F$ is not a fixed subfield of $K$ even though $F$ is topologically closed in $K$ and the algebraic closure of $F$ in $K$ is a multiquadratic extension of $F$ (cf., Proposition 6.1). A second example of this type appears in $\S 8$. In neither of the two examples is $F$ algebraically closed in $K$, and we know of no such examples. If there were such examples, however, they would have to have transcendence degree at least two, by Theorem 7.2 below. Theorem 7.2 is a corollary of the following computation of the group of $F$-automorphisms $\tau$ of $K$ with $v_{K} \tau=v_{K}$; we denote this group by $\operatorname{Aut}_{v}(K / F)$.

7.1 Proposition. Suppose $K / F$ is a field extension of transcendence degree one. Suppose $K$ is real Henselian and $F$ is algebraically and topologically closed in $K$. If we choose $y \in K^{\circ}$ and $A \subset K$ as indicated below, then there is a bijection $\Upsilon: \operatorname{Aut}_{v}(K / F) \rightarrow A$ with $\Upsilon(\tau)=\tau(y) / y$ for all $\tau \in \operatorname{Aut}_{v}(K / F)$. The set $A$ and element $y$ are chosen as follows:

(1) if $v K \neq v F$ and $v K \neq v\left(K^{2} F\right)$, then let $A=\sigma_{K}^{-1}\left(\mathbf{R}^{*}\right)$ and pick $y \in K$ with $v(y) \notin v\left(K^{2} F\right)$;

(2) if $v K \neq v F$ and $v K=v\left(K^{2} F\right)$, then let $A=\sigma_{K}^{-1}\left(\mathbf{R}^{\cdot 2}\right)$ and pick $y \in K$ with $v(y) \notin v F$

(3) if $v K=v F$ and $\bar{K} \neq \bar{F}$, then let $A=\sigma_{K}^{-1}(1)$ and pick $y \in \sigma_{K}^{-1}\left(\mathbf{R}^{\cdot 2}\right)$ with $\bar{y} \notin \bar{F}$;

(4) if $v K=v F$ and $\bar{K}=\bar{F}$, then pick $y \in \sigma_{K}^{-1}\left(\mathbf{R}^{\cdot 2}\right)$ with $y \notin F$ and let $A=1+I$ where $I=\{x \in K: v(x) \geq v(b-y)$ for all $b \in F\}$.

In the situation of (4) above, $y$ is the limit of a pseudoconvergent set in $F$ having no limit in $F$, and $I$ is simply the breadth ideal of this pseudoconvergent set [K, Theorem 1, and p. 304, Definitions]. The four cases in Proposition 7.1 are clearly exclusive and exhaustive.

ProOF. We first show $\Upsilon$ is injective; we only use here that $y \notin F$ and that $v(y) \notin v\left(K^{2} F\right)$ if $v K \neq v\left(K^{2} F\right)$. Suppose $\Upsilon(\delta)=\Upsilon(\tau)$ for $\delta, \tau \in \operatorname{Aut}_{v}(K / F)$. Let 
$\rho=\tau^{-1} \delta$. Then $y$ is in the fixed field $K^{\rho}$ of $\rho$. It suffices to show $K^{\rho}=K$, for then $\rho=1$, i.e., $\delta=\tau$. Just suppose $K^{\rho} \neq K$. Since $K / F$ has transcendence degree one, $K / K^{\rho}$ is algebraic, and hence multiquadratic (Proposition 6.1). Hence $K$ is a quadratic extension of a subfield $L$ containing $K^{\rho}$. Clearly $\bar{K}=\bar{L}$ (since $\bar{K}$ is real closed) and $L$ is real Henselian (Proposition 2.9), so $(v K: v L)=[K: L]=2[\mathbf{R}$, p. 236, Theorem 2 (Ostrowski)]. Since $v K / v F$ is a torsion-free group of rational rank at most one (Proposition 2.6 and [Bo, p. 166, Corollary 1]), $v K / v\left(F K^{2}\right)$ has at most two elements. By the choice of $y$ then, $v K=v\left(F(y) K^{2}\right)$. Thus $v K=v\left(L K^{2}\right)$. But this contradicts the assertion that $(v K: v L)=2$. We conclude that $\Upsilon$ is injective. It remains to compute its image.

We now verify that in each of our four cases, $\Upsilon$ maps surjectively onto $A$. Throughout the discussion below $\tau$ will denote an arbitrary element of $\operatorname{Aut}_{v}(K / F)$, $a$ will denote an arbitrary element of $A$, and $\theta: F[y] \rightarrow F[a y]$ will denote the ring homomorphism fixing $F$ and mapping $y$ to ay. We must show that $\Upsilon(\tau) \in A$ and that $\theta$ extends to an automorphism of $K$. By Theorem 3.5 it suffices to show ay is transcendental over $F$ (since then $\theta$ extends to an isomorphism $\theta_{1}: F(y) \rightarrow F(a y)$ and $K / F(a y)$ is algebraic) and that

$$
\begin{gathered}
v_{K} \theta=v_{K} \mid F[y] \quad\left(\text { so } v_{K} \theta_{1}=v_{K} \mid F(y)\right) \\
\sigma_{K} \theta_{1}=\sigma_{K} \mid F(y)
\end{gathered}
$$

and

$$
\theta\left(K^{2} \cap F[y]\right)=K^{2} \cap F[a y]
$$

(so that $\theta_{1}\left(K^{2} \cap F(y)\right)=K^{2} \cap F(a y)$ ).

CASE 1. $v K \neq v\left(K^{2} F\right)$. Since $\tau$ is an automorphism of valued fields, $\Upsilon(\tau)=$ $\tau(y) / y \in \sigma_{K}^{-1}\left(\mathbf{R}^{*}\right)$. Since $v(a y)=v(y)$ is not in the divisible hull of $v F$ (Proposition 2.6), ay is transcendental over $F$. Now let $b=\sum b_{i} y^{i} \in F[y]$ (with the obvious notation). Let $b_{t} y^{t}$ be the (unique) term of minimal value of $b$. Then [Bo, p. 161, Proposition 1]

$$
v(b)=v\left(b_{t} y^{t}\right)=v\left(b_{t}(a y)^{t}\right)=v(\theta(b)),
$$

proving (6). If $c=\sum c_{i} y^{i}$ has $v(c)=v(b)$ (so that $b / c$ is a typical unit of $F(y)$ ), then

$$
\sigma_{K}(b / c)=\sigma_{K}\left(b_{t} y^{t} / c_{t} y^{t}\right)=\sigma_{K}\left(b_{t}(a y)^{t} / c_{t}(a y)^{t}\right)=\sigma_{K}(\theta(b / c))
$$

proving (7). Also $b \in K^{2}$ if and only if $b_{t} y^{t} \in K^{2}$, and hence if and only if $t$ is even and $b_{t} \in K^{2}$ (using here that $y \notin v\left(K^{2} F\right)$ ). Similarly $\theta(b) \in K^{2}$ if and only if $t$ is even and $b_{t} \in K^{2}$. This proves (8) in this case.

Case 2. $v F \neq v K=v\left(K^{2} F\right)$. Then $v(y)=v\left(b^{2} c\right)$ for some $b \in K, c \in F$ and so $y=b^{2} c \varepsilon u^{2}$ where $\varepsilon \in \mathbf{Z}^{\cdot}$ and $u \in K$ (since $K$ is real Henselian). Thus

$$
\sigma_{K}(\Upsilon(\tau))=\sigma_{K}\left((\tau(b u) / b u)^{2}\right) \in \mathbf{R}^{\cdot 2}
$$

so $\Upsilon(\tau) \in A$. That ay is transcendental over $F$ and that equations (6) and (7) hold is proved just as in Case 1. Note that $a \in K^{\cdot 2}$. Suppose $b_{t} y^{t}$ is the term of $b=\sum b_{i} y^{i} \in F[y]$ of minimal value. Then $b \in K^{2}$ if and only if $b_{t} y^{t} \in K^{2}$, and hence if and only if $b_{t}(a y)^{t} \in K^{2}$, and thus if and only if

$$
\theta(b)=\sum b_{i}(a y)^{i} \in K^{2},
$$

proving (8). 
Case 3. $\bar{K} \neq \bar{F}$. Since $\bar{K}$ is Archimedean real closed, $\tau$ induces the identity map on $\bar{K}$. Thus $\tau(y)=u y$ where $\sigma_{K}(u)=1$. Hence $\Upsilon(\tau) \in \sigma_{K}^{-1}(1)$. Since $\bar{y}=\overline{a y}$ is transcendental over $\bar{F}$ (Proposition 2.6), then ay is transcendental over $F$. The valuation on $F[y]$ assigns to each $\sum b_{i} y^{i} \in F[y]$ the minimum of the values of the $b_{i}$ (e.g., see [Bo, p. 161, Proposition 2]). Thus $v_{K} \theta=v_{K} \mid F[y]$. That $\sigma_{K} \theta_{1}=\sigma_{K} \mid F(y)$ follows from the fact that $\theta_{1}$ induces the identity map on

$$
\overline{F(y)}=\bar{F}(\bar{y})=\bar{F}(\overline{a y})=\overline{\theta_{1}(F(y))}
$$

(identifying these rings with their canonical images in $\bar{K}$ ). Now suppose $f \in K^{2} \cap$ $F[y]$. Since $v K=v F$, there exists $b \in F$ with $v(f)=v\left(b^{2}\right)$. Thus $\sigma_{K}\left(f / b^{2}\right)>0$, so that $\sigma_{K}\left(\theta(f) / b^{2}\right)>0$. Thus $\theta(f)=b^{2}\left(\theta(f) / b^{2}\right) \in K^{2}$. Hence $\theta\left(K^{2} \cap F[y]\right) \subset$ $K^{2} \cap F[a y]$. The reverse inclusion follows by a similar argument.

Case 4. $K / F$ is an immediate extension of valued fields. Since $v \tau=v$, then

$$
v(\Upsilon(\tau)-1)=v(\tau(y-b)-(y-b)) \geq v(y-b)
$$

for all $b \in F$. Thus $\Upsilon(\tau)-1 \in I$, so $\Upsilon(\tau) \in A$. Next, $y$ is the limit of a pseudoconvergent set $Y$ in $F$ with no limit in $F$; $I$ is the breadth ideal of $Y[\mathbf{K}$, Theorem 1 and Definition, p. 304]. By [K, Lemma 3], $\theta(y)=a y$ is also a limit of $Y$, and hence [K, Theorem 2] $\theta$ extends to a homomorphism $\theta^{\prime}: F(y) \rightarrow F($ ay $)$ satisfying (6). (Note that $Y$ must be of transcendental type since otherwise by $[\mathbf{K}$, Theorem 3], $F$ has an immediate proper algebraic extension, contradicting Proposition 2.10.) In particular $a y$ is transcendental over $F$. Formula (7) follows from the fact that $K / F$ is immediate. Finally, (8) can be proved just as in Case 3. This completes the proof of 7.1.

7.2 THEOREM. Suppose $K$ is a real Henselian field, and $K / F$ is a field extension of transcendence degree one. Then $F$ is a fixed subfield of $K$ if $F$ is algebraically and topologically closed in $K$.

Proof. Let $w \in K \backslash F$. We must show that some $F$-automorphism of $K$ moves $w$. It suffices to show that $F(w)$ is not fixed by $\operatorname{Aut}_{v}(K / F)$. We apply the caseby-case analysis of automorphisms in Proposition 7.1.

Case 1: $v K \neq v\left(K^{2} F\right)$. Let $y \in K$ have $v(y) \notin v\left(K^{2} F\right)$. Then there exists $\tau \in \operatorname{Aut}_{v}(K / F)$ with $\tau(y)=2 y$. Since $K / F(w)$ is algebraic, then $v K / v(F(w))$ is torsion. Thus $v\left(y^{n}\right)=v(g)$ for some integer $n>0$ and some $g \in F(w)$. Thus $y^{n}=u g$ for some unit $u \in K$; we may assume $\sigma_{K}(u)>0$ (otherwise replace $u$ and $g$ by $-u$ and $-g$ ). Since $K$ is real Henselian, $u=c^{n}$ for some $c \in K$. Hence we may assume $u=1$ (replace $y$ by $y / c$ ). Thus $\tau(g)=2^{n} g \neq g$, as required.

Case 2. $v(K)=v\left(K^{2} \cap F\right) \neq v F$. Pick $y \in K$ with $v(y) \notin v F$. Now proceed just as in Case 1 .

Case 3. $\bar{K} \neq \bar{F}$. Since $K / F(w)$ is algebraic, then $\bar{K} / \overline{F(w)}$ is algebraic. But $\bar{F}$ is algebraically closed in $\bar{K}$ (Proposition 2.6). Hence there exists a unit $y \in F(w)$ with $\bar{y}$ transcendental over $\bar{F}$. But then by Proposition 7.1(3), $y$ is moved by some $F$-automorphism of $K$. (Note that $\sigma_{K}^{-1}(1)$ is not trivial since otherwise $F=\bar{F}$ is dense and topologically closed in $K=\bar{K}$, contradicting that $\bar{F} \neq \bar{K}$.)

Case 4. $K / F$ is immediate. There exists $a \in F$ with $\sigma_{K}(w / a)=1$. By 7.1(4), there is an $F$-automorphism of $K$ moving $w / a$. (Note that if we set $y=w / a$, then $I$ is nontrivial, since $F$ is closed in $K$ and $w / a \notin F$.) 
7.3 ExAmple. All the fields in this example will be subfields of the real Henselian field

$$
U:=\mathbf{R}\left(\left(x_{1}\right)\right)\left(\left(x_{2}\right)\right)\left(\left(x_{3}\right)\right)\left(\left(x_{4}\right)\right) .
$$

We let $F=F_{0}\left(\left(x_{4}\right)\right)$ where $F_{0}$ is the Henselization of $\mathbf{R}\left(x_{1}^{2}, x_{2}, x_{3}\right)$ in $U$ (Proposition $2.8)$. Set $y=b+x_{1} c$ where $b=\sum_{n>0}\left(x_{2} x_{3}^{2}\right)^{n !}$ and $c=\sum_{n>0}\left(x_{2} x_{3}\right)^{n !}$. Now let $K$ denote the Henselization of $F\left(x_{1}, y\right)$ in $U$. We will show below that $K$ is a real Henselian extension of $F$ of transcendence degree one. We will also show that the algebraic closure of $F$ in $K$ is a quadratic extension of $F$, namely $F\left[x_{1}\right]$, and that both $F$ and $F\left[x_{1}\right]$ are topologically closed in $K$. It follows from Theorem 7.2 that $F\left[x_{1}\right]$ is a fixed subfield of $K$. We will prove, however, that $F$ is not a fixed subfield of $K$.

Let $U_{0}=\mathbf{R}\left(\left(x_{1}\right)\right)\left(\left(x_{2}\right)\right)\left(\left(x_{3}\right)\right)$, so that $U=U_{0}\left(\left(x_{4}\right)\right)$. Let $v_{4}$ denote the $x_{4}$-adic valuation on $U$; then the canonical topology on $U$ is the valuation topology of $v_{4}$. Thus if the Laurent series $\sum a_{i} x_{4}^{i} \in U$ is in the closure of $F$, we then have for all $n>0$ an element $\sum b_{i} x_{4}^{i} \in F=F_{0}\left(\left(x_{4}\right)\right)$ with $v_{4}\left(\sum a_{i} x_{4}^{i}-\sum b_{i} x_{4}^{i}\right)>n$. Then $a_{i}=b_{i}$ for all $i<n$. Since this is true for all $n>0, a_{i} \in F_{0}$ for all $i$. Thus $\sum a_{i} x_{4}^{i} \in F$. Thus $F$ is topologically closed in $U$, and hence it is topologically closed in $K$. A similar argument shows that $F\left[x_{1}\right]=F_{0}\left[x_{1}\right]\left(\left(x_{4}\right)\right)$ is topologically closed in $K$.

Since $U / F\left[x_{1}\right]$ is an immediate extension of valued fields and $F\left[x_{1}\right]$ is real Henselian, then $F\left[x_{1}\right]$ has no proper algebraic extension in $U$ (Proposition 2.10). Thus $F\left[x_{1}\right]$ is the algebraic closure of $F$ in $K$.

To show $K$ has transcendence degree one over $F$, it suffices to show that $y$ is not algebraic over $F_{0}$. Suppose the contrary. There is an $\mathbf{R}\left(\left(x_{1}^{2}\right)\right)\left(\left(x_{2}\right)\right)\left(\left(x_{3}\right)\right)$ automorphism $\theta$ of $U_{0}$ with $\theta\left(x_{1}\right)=-x_{1}$. Then $b=(y+\theta(y)) / 2$ is algebraic over $F_{0}$, and hence over $\mathbf{R}\left(x_{1}^{2}, x_{3}\right)\left(x_{2} x_{3}^{2}\right)$, contradicting [Bo, p. 173, Exercise 1a]. Hence the field extension $K / F$ has transcendence degree one. (This fact is also an immediate corollary of the Claim below.)

It remains to show that $F$ is not a fixed subfield of $K$. Some notation will be helpful. We let $v: U \rightarrow(\mathbf{Z} \times \mathbf{Z} \times \mathbf{Z} \times \mathbf{Z}) \cup\{\infty\}$ denote the canonical valuation on $U$; $v\left(x_{i}\right)$ is the 4-tuple with all zeros except for a 1 in the $i$ th position from the right (for $i \leq 4)$. We let $v_{0}$ be the canonical valuation on $U_{0}$. Let $K_{0}$ denote the Henselization of $F_{0}\left(x_{1}, y\right)$ in $U$. By Proposition 2.8, $K_{0} \subset K$ (since $\left.F_{0}\left(x_{1}, y\right) \subset F\left(x_{1}, y\right)\right)$ and $K \subset K_{0}\left(\left(x_{4}\right)\right)$ (since $\left.F\left(x_{1}, y\right)=F_{0}\left(\left(x_{4}\right)\right)\left(x_{1}, y\right) \subset K_{0}\left(\left(x_{4}\right)\right)\right)$.

Just suppose $F$ is a fixed subfield of $K$. Then there exists $\tau \in \operatorname{Aut}(K / F)$ with $\tau\left(x_{1}\right)=-x_{1}$. For each positive integer $n$, let

$$
y_{n}=\sum_{1 \leq i<n} x_{1}\left(x_{2} x_{3}\right)^{i !}+\left(x_{2} x_{3}^{2}\right)^{i !} .
$$

Note that

$$
v\left(y-y_{n}\right)=v\left(x_{1}\left(x_{2} x_{3}\right)^{n !}+\left(x_{2} x_{3}^{2}\right)^{n !}\right)=(0, n !, n !, 1)=v\left(y_{n+1}-y_{n}\right)
$$

Thus $\left\{y_{n}\right\}_{n>1}$ is a pseudoconvergent set with limit $y$. Thus $\tau(y)$ must be a limit of the pseudoconvergent set

$$
\left\{\tau\left(y_{n}\right)\right\}_{n>1}=\left\{\sum_{1 \leq i<n}\left(x_{2} x_{3}^{2}\right)^{i !}-x_{1}\left(x_{2} x_{3}\right)^{i !}\right\}_{n>1} .
$$


( $v \tau=v$ since $\tau$ induces an automorphism of $v K$ which fixes $v F$, and hence is the identity map.) Let $z=\tau(y)+y$. Then for all $n>1$,

$$
v(z-2 b)=v\left(\tau(y)-\tau\left(y_{n}\right)+y-y_{n}-2 \sum_{n \leq i}\left(x_{2} x_{3}^{2}\right)^{i !}\right) \geq(0, n !, n !, 1) .
$$

Since $v(y)>0$, then $v(\tau(y))>0$ and so $v_{4}(y) \geq 0$ and $v_{4}(\tau(y)) \geq 0$, so $v_{4}(z) \geq 0$. Hence we can write $z=\sum_{i \geq 0} b_{i} x_{4}^{i}$ where $b_{i} \in K_{0}$ for all $i \geq 0$. Then for all $n>1$,

$$
v\left(b_{0}-2 b\right)=v\left(z-2 b-\sum_{i>0} b_{i} x_{4}^{i}\right)>(0, n !, 0,0) .
$$

Thus $v_{0}\left(b_{0}-2 b\right)=\infty$, and so $b=b_{0} / 2 \in K_{0}$. Hence both $b$ and $c$ are in $K_{0}$, which is an extension of $\mathbf{R}\left(x_{1}, x_{2}, x_{3}\right)$ of transcendence degree at most 1 . This contradicts the next Claim (take $E=\mathbf{R}\left(x_{1}\right), x=x_{2}, y=x_{3}$ ), whose proof will therefore complete this example.

Claim. Let $E$ be a field. Then the elements $b=\sum_{n>0}\left(x y^{2}\right)^{n !}$ and $c=$ $\sum_{n>0}(x y)^{n !}$ of the iterated Laurent series field $E((x))((y))$ are algebraically independent over the rational function field $E(x, y)$.

PROOF OF Claim. Let $v: E((x))((y)) \rightarrow \mathbf{Z} \times \mathbf{Z} \cup\{\infty\}$ be the canonical valuation. Suppose $b$ and $c$ are not algebraically independent over $E(x, y)$. Then there exists a nonempty finite set $I$ of 4 -tuples of nonnegative integers and elements $a_{i} \in E^{\cdot}$ for each $i \in I$, such that $0=\sum_{i \in I} a_{i} x^{i_{1}} y^{i_{2}} b^{i_{3}} c^{i_{4}}$ (where for $i \in I$ we write $\left.i=\left(i_{1}, i_{2}, i_{3}, i_{4}\right)\right)$. Let $D_{t}=\max _{i \in I} i_{t}$, for $t=1,2,3,4$. Pick $n>2\left(D_{1}+D_{2}+D_{3}+D_{4}\right)$. Also write $b_{n}=\sum_{0<t \leq n}\left(x y^{2}\right)^{t !}$ and $b_{n}^{\prime}=b-b_{n}$, and $c_{n}=\sum_{0<t \leq n}(x y)^{t !}$ and $c_{n}^{\prime}=c-c_{n}$. Then

$$
0=\sum_{i \in I} a_{i} x^{i_{1}} y^{i_{2}}\left(b_{n}+b_{n}^{\prime}\right)^{i_{3}}\left(c_{n}+c_{n}^{\prime}\right)^{i_{4}}=\sum_{i \in I} a_{i} x^{i_{1}} y^{i_{2}} b_{n}^{i_{3}} c_{n}^{i_{4}}+\Delta
$$

where $\Delta$ is a formal sum of monomials of the form $a x^{m} y^{t}(a \in E)$ of value at least $(n+1) !(1,1)$. On the other hand $\sum_{i \in I} a_{i} x^{i_{1}} y^{i_{2}} b_{n}^{i_{3}} c_{n}^{i_{4}}$ is finite sum of monomials $a x^{m} y^{t}$ of value at most

$$
v\left(x^{D_{1}} y^{D_{2}}\left(x y^{2}\right)^{n ! D_{3}}(x y)^{n ! D_{4}}\right)=\left(D_{2}+n !\left(2 D_{3}+D_{4}\right), D_{1}+n !\left(D_{3}+D_{4}\right)\right)
$$

which is strictly less than $(n+1) !(1,1)$ by choice of $n$. Thus

$$
\sum_{i \in I} a_{i} x^{i_{1}} y^{i_{2}} b_{n}^{i_{3}} c_{n}^{i_{4}}=0 .
$$

For each $i \in I, a_{i} x^{i_{1}} y^{i_{2}} b_{n}^{i_{3}} c_{n}^{i_{4}}$ is a sum of monomials $a x^{m} y^{t}$ and exactly one of the monomials has maximal value, namely $a_{i} x^{i_{1}} y^{i_{2}}\left(x y^{2}\right)^{n ! i_{3}}(x y)^{n ! i_{4}}$, which has value

$$
\left(i_{2}+n !\left(2 i_{3}+i_{4}\right), i_{1}+n !\left(i_{3}+i_{4}\right)\right) .
$$

Now pick $i \in I$ with the expression (10) as large as possible. Because of (9), there must also exist $j \in I, j \neq i$, with $\left(j_{2}+n !\left(2 j_{3}+j_{4}\right), j_{1}+n !\left(j_{3}+j_{4}\right)\right)$ equal to the quantity in (10). Thus

$$
i_{2}+n !\left(2 i_{3}+i_{4}\right)=j_{2}+n !\left(2 j_{3}+j_{4}\right)
$$

so $n$ ! divides $i_{2}-j_{2}$. Thus $i_{2}=j_{2}$ (note $\left|i_{2}-j_{2}\right|<n$ ). Hence $2 j_{3}+j_{4}=2 i_{3}+i_{4}$. Similarly $i_{1}=j_{1}$ and $i_{3}+i_{4}=j_{3}+j_{4}$. Thus $i=j$, a contradiction. This completes the proof of the above Claim. 
8. An example. Let $G$ denote the direct sum $\cdots \mathbf{Z} \oplus \mathbf{Z} \oplus \mathbf{Z}$; give $G$ the lexicographic order. $G$ is an ordered abelian group. Formally we regard $G$ as the set of all maps $f:-\mathbf{N} \rightarrow \mathbf{Z}$ with finite support, where $-\mathbf{N}$ denotes the set of negative integers. If $f, g \in G$, then $f+g$ is defined pointwise (i.e., $(f+g)(m)=f(m)+g(m)$ for all $m \in-\mathbf{N})$ and we write $f \leq g$ if $f(m) \leq g(m)$ where $m$ is the minimum of $\{i \in-\mathbf{N}: f(i) \neq 0$ or $g(i) \neq 0\}$. For each $i \in \mathbf{N}$ we let $\varepsilon_{i} \in G$ be the map with $\varepsilon_{i}(k)=1$ if $k=-i$ and $\varepsilon_{i}(k)=0$ if $k \neq-i$. Thus $\varepsilon_{i}$ can be regarded as the "(-N)-tuple" $\left(\ldots, a_{3}, a_{2}, a_{1}\right)$ consisting of all zeros except for $a_{i}$, which has the value 1 .

Now let $K=\mathbf{R}\left(\left(t^{G}\right)\right)$ and $F=\mathbf{R}\left(\left(t^{2 G}\right)\right)$. Formally, the generalized Laurent series field $K$ consists of all maps $G \rightarrow \mathbf{R}$ with well-ordered support. We think of elements $f$ of $K$ as formal series $\sum_{\gamma \in G} f(\gamma) t^{\gamma}$. Addition is pointwise $\left(\sum a_{\gamma} t^{\gamma}+\right.$ $\left.\sum b_{\gamma} t^{\gamma}=\sum\left(a_{\gamma}+b_{\gamma}\right) t^{\gamma}\right)$ and multiplication is by Cauchy product:

$$
\left(\sum a_{\gamma} t^{\gamma}\right)\left(\sum b_{\gamma} t^{\gamma}\right)=\sum_{\gamma}\left(\sum_{\rho+\delta=\gamma} a_{\rho} b_{\delta}\right) t^{\gamma} .
$$

$K$ is a maximal valued field with residue class field $\mathbf{R}$; the valuation $v_{K}$ assigns to each element $f: G \rightarrow \mathbf{R}$ in $K^{*}$ the minimum of its support. (See the references at the end of $\S 1$.) We regard $F$ as a subfield of $K$ in the obvious way.

Let $E$ denote the algebraic closure of $F$ in $K$. For each $i \in \mathbf{N}$ let $t_{i}=t^{\varepsilon_{i}}$, so $v\left(t_{i}\right)=\varepsilon_{i}$. (Formally, $t_{i}$ is the map $G \rightarrow \mathbf{R}$ which is zero on $G$ except at $\varepsilon_{i}$, where it takes the value 1.) Since the $\varepsilon_{i}$ form a basis for $G$ we can regard each $f \in K$ as a formal sum

$$
\sum_{\varepsilon \in G} f(\varepsilon) \prod_{i=1}^{\infty} t_{i}^{\varepsilon(-i)}
$$

where each of the products in (11) has only finitely many nontrivial (i.e., $\neq 1$ ) factors and the terms of the sum are zero except on a well-ordered subset of $G$.

8.1 Proposition. $E=F\left[t_{1}, t_{2}, t_{3}, \ldots\right]$.

Proof. Let $E^{\prime}=F\left[t_{1}, t_{2}, t_{3}, \ldots\right]$. Then $E^{\prime} \subset E$ since $t_{i}^{2}=t^{2 \varepsilon_{i}} \in F$ for all $i$. Since $F$ is a Henselian valued field, then so is $E^{\prime}$. Further, $v E^{\prime}=G=v E$ (the $\varepsilon_{i}$ generate $G$ ) and $\overline{E^{\prime}}=\mathbf{R}=\bar{E}$. Thus $E$ is an immediate algebraic extension of the real Henselian field $E^{\prime}$. Thus $E=E^{\prime}$ (Proposition 2.10).

\subsection{Proposition. $E$ is dense in $K$.}

ProOF. Let $\alpha \in K$; we prove that $\alpha$ is in the closure of $E$. Let $n \in \mathbf{N}$. Without loss of generality, $\alpha$ is a unit (since if $t^{-v(\alpha)} \alpha$ is in the closure of $E$, then so is $\alpha$ ). We can write $\alpha=\alpha_{0}+\alpha_{1}$ where $\alpha_{0}(\varepsilon)=\alpha(\varepsilon)$ for all $\varepsilon \in G$ with $\varepsilon(-i)=0$ for all $i>n$ and $\alpha_{0}(\varepsilon)=0$ otherwise. Less formally, $\alpha_{0}$ is the sum of all monomial summands of $\alpha$ of the form $a t_{1}^{r_{1}} \cdots t_{n}^{r_{n}}$ and $\alpha_{1}$ is a sum of monomials of the form $a t_{1}^{r_{1}} \cdots t_{m}^{r_{m}}$ where $m>n$ and (since $v(\alpha)=0$ ) also $r_{m}>0$. Thus

$$
v\left(\alpha-\alpha_{0}\right)=v\left(\alpha_{1}\right)>\varepsilon_{n} .
$$

Also, $\alpha_{0} \in \mathbf{R}\left(\left(t_{1}\right)\right) \cdots\left(\left(t_{n}\right)\right) \subset E$. Since the set $\left\{\varepsilon_{n}\right\}_{n>0}$ is cofinal in $G$, this shows $\alpha$ is in the closure of $E$. 


\subsection{COROllary. $\operatorname{Aut}(K / E)=1$.}

ProOF. Apply Propositions 6.1 and 8.2.

8.4 COROLlaRY. There is a group isomorphism $\Psi: \operatorname{Aut}(K / F) \rightarrow\left(\mathbf{Z}^{*}\right)^{\mathbf{N}}$ with $\Psi(\tau)=\left(\tau\left(t_{i}\right) / t_{i}\right)_{i \in \mathbf{N}}$ for each $\tau \in \operatorname{Aut}(K / F)$.

PROOF. We use Remark 4.6. $\operatorname{Aut}_{v F}(v K)=1$ since $v K / v F=G / 2 G$ is torsion. Hence $\operatorname{Aut}_{v}(K / F)=\operatorname{Aut}(K / F)$. Now suppose $\tau \in \operatorname{Aut}(K / F)$ is an order automorphism, say for an ordering $P$ of $K$. Then $\tau \mid E=1$ (since $\tau \mid E \in \operatorname{Aut}(E / F)$; now apply the uniqueness part of Theorem 3.1). Hence $\tau=1$ (Corollary 8.3). Thus the map $\Phi: \operatorname{Aut}(K / F) \rightarrow \operatorname{Hom}\left(v K / v F, \mathbf{Z}^{*}\right)$ is an isomorphism (Remark 4.6). The map $\Psi$ is the composition of $\Phi$ with the isomorphism $\Phi^{\prime}: \operatorname{Hom}\left(G / 2 G, \mathbf{Z}^{*}\right) \rightarrow\left(\mathbf{Z}^{*}\right)^{\mathbf{N}}$ associated with the basis $\left(t_{i}+2 G\right)_{i \in \mathbf{N}}$ of $G / 2 G$ (that is, $\Phi^{\prime}(f)=\left(f\left(t_{i}+2 G\right)\right)_{i \in \mathbf{N}}$ for any $\left.f \in \operatorname{Hom}\left(G / 2 G, \mathbf{Z}^{*}\right)\right)$.

For any $I \in\left(\mathbf{Z}^{*}\right)^{\mathbf{N}}$ we let $\sigma_{I}=\Psi^{-1}(I)$. Then for any $f \in K$

$$
\sigma_{I}(f)(\varepsilon)=f(\varepsilon) \prod_{i \in \mathbf{N}} I(i)^{\varepsilon(-i)}
$$

for all $\varepsilon \in G$. In terms of formal series,

$$
\sigma_{I}\left(\sum_{\varepsilon \in G} f(\varepsilon) \prod_{i \in \mathbf{N}} t_{i}^{\varepsilon(-i)}\right)=\sum_{\varepsilon \in G} f(\varepsilon) \prod_{i \in \mathbf{N}}\left(I(i) t_{i}\right)^{\varepsilon(-i)}
$$

(cf., formula (11)).

To establish our counterexample to the converse of Proposition 6.1 (and to the generalization of Theorem 6.3) we need some information about sets of elements of $K$ which are algebraically independent over $E$. In general little seems known about algebraic independence of elements of the maximal immediate extension of a field over the field. (The Claim of $\S 7$ was a result about this situation.)

8.5 Proposition. Let $\left\{N_{\beta}: \beta \in B\right\}$ be a set of pairwise disjoint nonempty infinite subsets of $\mathbf{N}$. For each $\beta \in B$, let $r_{\beta}=\sum_{i \in N_{\beta}} t_{i}$. Then the set $\left\{r_{\beta}: \beta \in B\right\}$ is algebraically independent over $F$.

Thus, for example, $t_{1}+t_{3}+t_{5}+\cdots$ and $t_{2}+t_{4}+t_{6}+\cdots$ are algebraically independent over $F$.

PROOF. Pick $\beta \in B$. Let $H$ be the group of elements of Aut $(K / F)$ fixing every element of $\left\{t_{i}: i \in \mathbf{N}\right\}$ except for a finite subset of $\left\{t_{i}: i \in \mathbf{N}_{\beta}\right\}$. Then for each $\alpha \in B, r_{\alpha} \in K^{H}$ if and only if $\alpha \neq \beta$. Let $L$ denote the algebraic closure of $K^{H}$ in $K$. $L$ is an algebraic extension of $E K^{H}$. Since $K^{H}$ is real Henselian (Proposition 2.9), so is its algebraic extension $E K^{H}$. Also $L / E K^{H}$ is an immediate extension (since $K / E$ is immediate). Thus $L=E K^{H}$ by Proposition 2.10. Consider now the field $L^{\prime}=K^{H}\left[t_{r(1)}, \ldots, t_{r(n)}\right]$ where $r(1), \ldots, r(n)$ is any finite sequence of elements of $N_{\beta}$. Elements of $K^{H}$ are formal linear combinations of monomials $t_{1}^{m_{1}} t_{2}^{m_{2}} \cdots t_{s}^{m_{s}}$ where the integers $m_{i}$ are even if $i \in N_{\beta}$ (cf., Proposition 8.4). The set of square-free products of $t_{r(1)}, \ldots, t_{r(n)}$ spans $L^{\prime}$ as a $K^{H}$-vector space. Hence for all choices of the $r(i), r_{\beta}=\sum_{i \in N_{\beta}} t_{i}$ is clearly not in $L^{\prime}$. Hence $r_{\beta} \notin L$. Thus $r_{\beta}$ is transcendental over $K^{H}$, and hence over $F\left(\left\{r_{\alpha}: \alpha \neq \beta\right\}\right)$. Since $\beta \in B$ was chosen arbitrarily, this shows $\left\{r_{\alpha}: \alpha \in B\right\}$ is algebraically independent over $F$. 
8.6 REMARK. One consequence of 8.5 is that $E \neq K$. Thus $E$ is not a fixed subfield of $K$ (cf., Corollary 8.3). However $F$ is a fixed subfield of $K$. (Use Proposition 8.1 to apply Theorem 6.3. $F$ is closed in $K$ since the valuation topology on $K$ induces that on $F$, and $F$ is topologically complete.) Thus we have the perhaps unexpected result that the algebraic closure in $K$ of a fixed subfield of $K$ is not necessarily itself a fixed subfield of $K$.

We are now ready to construct a counterexample to the converse of Proposition 6.1 .

8.7 EXAMPLE. Let $e=t_{2}+t_{4}+t_{6}+\cdots$ and $n=t_{3}+t_{5}+t_{7}+\cdots$. Let $\alpha=\left(1+t_{1}\right) e+n$. Let $L$ be a real Henselization of $E(\alpha)$ contained in $K$. Then $L$ is real Henselian, $F$ is topologically closed in $L$, and the algebraic closure of $F$ in $L$ (namely, $E$ ) is a multiquadratic extension of $F$. However, $F$ is not a fixed subfield of $L$.

PROOF. $L$ is well defined by Proposition 2.8. Proposition 8.1 tells us that the algebraic closure of $F$ in $L$ is a multiquadratic extension of $F$. That $F$ is closed in $L$ follows from the same argument that was used in Remark 8.6 above to show $F$ is closed in $K$.

Now suppose $F$ is a fixed subfield of $L$. Then some $F$-automorphism $\tau$ of $L$ moves $t_{1}$. By $[\mathbf{K}$, Theorem 5], $\tau$ extends to an automorphism of $K$. Thus there exists $I \in$ $\left(\mathbf{Z}^{*}\right)^{\mathbf{N}}$ with $\tau=\sigma_{I} \mid L$ (Proposition 8.4). Let $D=I^{-1}(-1) \backslash\{1\} ; D=\{i \in \mathbf{N}: i>1$ and $\left.\tau\left(t_{i}\right)=-t_{i}\right\}$. Also set $\alpha_{n}=\sum_{i \in D \backslash 2 \mathbf{N}} t_{i}, \alpha_{e}=\sum_{i \in D \cap 2 \mathbf{N}} t_{i}, \alpha_{n}^{\prime}=n-\alpha_{n}$ and $\alpha_{e}^{\prime}=e-\alpha_{e}$. Thus $\alpha_{n}^{\prime}$ is the sum of the $t_{i}$ where $i$ is odd, larger than 1 , and not in $D$ and $\alpha_{e}^{\prime}$ is the sum of the $t_{i}$ where $i$ is even and not in $D$. Then $\alpha=\left(\alpha_{n}+\alpha_{n}^{\prime}\right)+\left(1+t_{1}\right)\left(\alpha_{e}+\alpha_{e}^{\prime}\right)$, so that $\tau(\alpha)=-\alpha_{n}+\alpha_{n}^{\prime}+\left(1-t_{1}\right)\left(-\alpha_{e}+\alpha_{e}^{\prime}\right)$. Thus

$$
\alpha_{n}^{\prime}+\alpha_{e}^{\prime}+t_{1} \alpha_{e}=(\alpha+\tau(\alpha)) / 2 \in L
$$

and

$$
\alpha_{n}+\alpha_{e}+t_{1} \alpha_{e}^{\prime}=(\alpha-\tau(\alpha)) / 2 \in L .
$$

Let us call a formal series $\beta \in \mathbf{R}\left(\left(t^{G}\right)\right)$ "finite" if it is a finite linear combination of products of the $t_{i}$. Thus either $\alpha_{e}$ or $\alpha_{e}^{\prime}$ is not finite and similarly for either $\alpha_{n}$ or $\alpha_{n}^{\prime}$. If none of $\alpha_{n}, \alpha_{n}^{\prime}, \alpha_{e}$ and $\alpha_{e}^{\prime}$ are finite then by Proposition 8.5 all four are algebraically independent over $F$. But all four are in $L\left(\alpha_{e}, \alpha_{e}^{\prime}\right)$, which has transcendence degree at most three over $F$. Thus at least one of $\alpha_{n}, \alpha_{n}^{\prime}, \alpha_{e}, \alpha_{e}^{\prime}$ is finite. If $\alpha_{n}$ is finite and the rest are not, then all four are in $L\left(\alpha_{e}\right)$. But this is impossible since $L\left(\alpha_{e}\right)$ has transcendence degree at most 2 over $F$ but $\alpha_{n}^{\prime}, \alpha_{e}$ and $\alpha_{e}^{\prime}$ are algebraically independent over $F$ (Proposition 8.5). Similarly one argues neither $\alpha_{n}^{\prime}, \alpha_{e}$ nor $\alpha_{e}^{\prime}$ can be the only finite element among the four. Thus we conclude that exactly two of the elements $\alpha_{n}, \alpha_{n}^{\prime}, \alpha_{e}, \alpha_{e}^{\prime}$ are finite. But then all of them lie in $L$ (again apply formulas (12) and (13)), even though two are algebraically independent over $F$. This is impossible. Thus $F$ is not a fixed subfield of $L$.

We have the usual bijective Galois correspondence between the fixed subfields of $K$ containing $F$ and the groups of automorphisms of $K$ of the form $\operatorname{Aut}(K / S)$ where $K \supset S \supset F$. The fixed subfields of $K$ containing $F$ can be intrinsically characterized as the subfields of $K$ containing $F$ which are topologically closed in $K$ and which have relative algebraic closures in $K$ which are multiquadratic extensions 
of themselves (Theorem 6.3). We next show that the groups of automorphisms of the form $\operatorname{Aut}(K / S)$ where $K \supset S \supset F$ are exactly the closed subgroups of $\operatorname{Aut}(K / F)$ where we give $\operatorname{Aut}(K / F)$ the compact open topology (cf., the proof of 8.8 below), regarding Aut $(K / F)$ as a set of continuous functions $K \rightarrow K$ where $K$ has its usual topology (cf., the first paragraph of $\S 6)$. This topology on $\operatorname{Aut}(K / F)$ is not the Krull topology (see Example 8.9 below).

8.8 Proposition. Let $G$ be a subgroup of $\operatorname{Aut}(K / F)$. Then $\operatorname{Aut}\left(K / K^{G}\right)$ is the closure of $G$ in the compact open topology (where $K$ has the valuation topology).

ProOF. It is easy to show that $\operatorname{Aut}\left(K / K^{G}\right)$ is closed in the compact open topology on $\operatorname{Aut}(K / F)$ for any Hausdorff topological field $K$. We now show $G$ is dense in $\operatorname{Aut}\left(K / K^{G}\right)$ for our particular field $K$. Let $C_{1}, \ldots, C_{r}$ be compact subsets of $K$ and $U_{1}, \ldots, U_{r}$ be open subsets of $K$. Let $W$ denote the set of $\rho \in \operatorname{Aut}(K / F)$ with $\rho\left(C_{i}\right) \subset U_{i}$ for $i=1, \ldots, r$. Then $W$ is a typical basic open set in $\operatorname{Aut}(K / F)$. Let $\tau \in W \cap \operatorname{Aut}\left(K / K^{G}\right)$. It suffices to show $W \cap G$ is nonempty.

For each $n \in \mathbf{N}$ let $B_{n}=\left\{b \in K: v(b)>\varepsilon_{n}\right\}$ and $B_{n}^{\prime}=\left\{b \in K: v(b)>-\varepsilon_{n}\right\}$. Since the $B_{n}^{\prime}$ cover $K$, there exists $N$ with $B_{N}^{\prime} \supset C_{i}$ for all $i \leq r$. Now let us fix some $i, 1 \leq i \leq r$. For each $n \in \mathbf{N}$ let $W_{i}(n)$ denote the union of all sets of the form $\tau(a)+B_{n}$ where $a \in C_{i}$ and $\tau(a)+B_{n} \subset U_{i}$. The sets $W_{i}(n), n \in \mathrm{N}$, cover the compact set $\tau\left(C_{i}\right)$. Since $B_{n}$ is an additive group, $\tau\left(C_{i}\right) \cap W_{i}(n) \subset W_{i}(n+1)$ for all $n \in \mathbf{N}$. (After all if $b \in C_{i}$ and $\tau(b) \in W_{i}(n)$, then for some $a \in C_{i}, \tau(b) \in$ $\tau(a)+B_{n} \subset U_{i}$. Then

$$
\tau(b) \in \tau(b)+B_{n+1} \subset \tau(b)+B_{n}=\tau(a)+B_{n} \subset U_{i},
$$

whence $\tau(b) \in W_{i}(n+1)$.) Hence $\tau\left(C_{i}\right) \subset W_{i}\left(N_{i}\right)$ for some $N_{i}$. Thus we can pick $N^{\prime}$ with $\tau\left(C_{i}\right) \subset W_{i}\left(N^{\prime}\right)$ for all $i \leq r$. Let $M=N+N^{\prime}$.

The elements of $G$ induce $F$-automorphisms of $E$; let $G^{\prime}=\{\rho \mid E: \rho \in G\}$. Clearly $E^{G^{\prime}}=E \cap K^{G}$. Thus $\tau \mid E$ fixes $E^{G^{\prime}}$, so $\tau \mid E$ is in the closure of $G^{\prime}$ in $\operatorname{Aut}(E / F)$ in the Krull topology [S, Appendix I]. Hence there exists $\rho \in G$ such that $\rho$ and $\tau$ agree on the subset $\left\{t_{1}, \ldots, t_{M}\right\}$ of $E$. Now let $i \leq r$. Pick $d \in C_{i}$. It suffices to show $\rho(d) \in U_{i}$. (If this is true for all $i$, then $\rho \in W \cap G$, so $W \cap G$ is nonempty, as required.)

Write $d=\sum_{\varepsilon \in G} d(\varepsilon) t^{\varepsilon}$, where for each $\varepsilon \in G, t^{\varepsilon}=\prod_{i=1}^{\infty} t_{i}^{\varepsilon(-i)}$ (cf., formula (11)). Note $\rho\left(t^{\varepsilon}\right)-\tau\left(t^{\varepsilon}\right)=0$ if $\varepsilon(-i)=0$ for all $i>M$. Thus $\rho(d)-\tau(d)$ is a sum of monomials $d(\varepsilon) t^{\varepsilon}$ where $d(\varepsilon) \neq 0$ and $\varepsilon(-i) \neq 0$ for some $i>M$. Consider any such $\varepsilon=\varepsilon^{*}$. Let $j$ be maximal with $\varepsilon^{*}(-j) \neq 0$. Just suppose $\varepsilon^{*}(-j)<0$. Then $v\left(d\left(\varepsilon^{*}\right) t^{\varepsilon^{*}}\right)<-\varepsilon_{j-1} \leq-\varepsilon_{M}$. But since $C_{i} \subset B_{M}^{\prime},-\varepsilon_{M}<$ $v(d)=\min \{\varepsilon: d(\varepsilon) \neq 0\} \leq \varepsilon^{*} \leq-\varepsilon_{M}$, a contradiction. Hence $\varepsilon^{*}(-j)>0$. Thus $v\left(d\left(\varepsilon^{*}\right) t^{\varepsilon^{*}}\right)>\varepsilon_{j-1} \geq \varepsilon_{M}$. Thus $v(\rho(d)-\tau(d))>\varepsilon_{M}$. Hence $\rho(d) \in \tau(d)+B_{M}$. Since $\tau\left(C_{i}\right) \subset W_{i}(M), \tau(d) \in \tau(a)+B_{M} \subset U_{i}$ for some $a \in C_{i}$. Thus

$$
\rho(d) \in \tau(d)+B_{M}=\tau(a)+B_{M} \subset U_{i},
$$

as required.

8.9 ExAmple. Let $A$ be the subgroup of $\operatorname{Aut}(K / F)$ consisting of all $\tau \in$ $\operatorname{Aut}(K / F)$ which fix all but a finite subset of $\left\{t_{i}: i \in \mathbf{N}\right\}$. Then $K^{A}=F$ (Proposition 8.4), so $A$ is dense in $\operatorname{Aut}(K / F)$ where $\operatorname{Aut}(K / F)$ has the topology of Proposition 8.8. Now give $\operatorname{Aut}(K / F)$ the Krull topology, i.e., the compact open topology 
where $K$ is regarded as a discrete space. Then $A$ is closed in $\operatorname{Aut}(K / F)$, so that $\operatorname{Aut}\left(K / K^{A}\right)$ is not the closure of $A$. For suppose $I \in\left(\mathbf{Z}^{\cdot}\right)^{\mathbf{N}}$. If $\sigma_{I} \notin A$, then $\sigma_{I}^{-1}(-1)$ is infinite, so that no element of $A$ can agree with $\sigma_{I}$ on $\sum_{i \in \mathrm{N}} t_{i}$. Thus $\sigma_{I}$ is not in the closure of $A$. Hence $A$ is closed in $\operatorname{Aut}(K / F)$, as claimed.

The above observations show that the topology on $\operatorname{Aut}(K / F)$ in Proposition 8.8 is not the Krull topology. (For the use of the Krull topology in the Galois theory of infinite algebraic extensions see [S, Appendix I].)

\section{REFERENCES}

[AS] E. Artin and O. Schreier, Algebraische Konstruktion reeller Körper, Abh. Math. Sem. Univ. Hamburg 5 (1927), 85-99.

[B] E. Becker, Extended Artin-Schreier theory of fields, Rocky Mountain J. Math. 14 (1984), 881897.

[BHR] E. Becker, J. Harman, and A. Rosenberg, Signatures of fields and extension theory, J. Reine Angew. Math. 330 (1982), 53-75.

[Bo] N. Bourbaki, Éléments de mathématique. Fasc. XXX. Algèbre commutative, Chapitre 6: Valuations, Actualités Sci. Indust., no. 1308, Hermann, Paris, 1964.

[Br1] R. Brown, Real places and ordered fields, Rocky Mountain J. Math. 1 (1971), 633-636.

[Br2] _ An approximation theorem for extended prime spots, Canad. J. Math. 24 (1972), 167184.

[Br3] _ Extended prime spots and quadratic forms, Pacific J. Math. 51 (1974), 379-395.

[Br4] _ Superpythagorean fields, J. Algebra 42 (1976), 483-494.

[Br5] _ Real closures of fields at orderings of higher level, Pacific J. Math. 127 (1987), 261-279.

[Br6] _ The behavior of chains of orderings under field extensions and places, Pacific J. Math. 127 (1987), 281-297.

[Br7] _ Orderings and order closures of not necessarily formally real fields, in preparation.

[BCP] R. Brown, T. Craven, and M. J. Pelling, Ordered fields satisfying Rolle's Theorem, Illinois J. Math. 30 (1986), 66-78.

[E] O. Endler, Valuation theory, Springer-Verlag, New York, 1972.

[H] J. Harman, Chains of higher level orderings, Ph.D. Dissertation, Univ. of California, Berkeley, 1980.

[HW] D. Harrison and H. Warner, Infinite primes of fields and completions, Pacific J. Math. 45 (1973), 201-206.

[K] I. Kaplansky, Maximal fields with valuations, Duke Math. J. 9 (1942), 303-321.

[L] T. Y. Lam, Algebraic theory of quadratic forms, Benjamin/Cummings, Reading, Mass., 1973.

[PR] A. Prestel and P. Roquette, Formally p-adic fields, Lecture Notes in Math., vol. 1050, Springer-Verlag, Berlin, 1984.

[R] P. Ribenboim, Théorie des valuations, 2ième éd., Sém. Math. Supérieures, no. 9, Presses Univ. Montréal, Montréal, Que., 1968.

[S] O. F. G. Schilling, The theory of valuations, Math. Surveys, no. 4, Amer. Math. Soc., Providence, R. I., 1950.

[V] K. Valente, The p-primes of a commutative ring, Pacific J. Math. 126 (1987), 385-400.

Department of Mathematics, University of HaWail, Honolulu, haWail 96822 